\title{
Joint Precoding and Multivariate Backhaul Compression for the Downlink of
} Cloud Radio Access Networks

\author{
Seok-Hwan Park, Osvaldo Simeone, Onur Sahin and Shlomo Shamai (Shitz)
}

\begin{abstract}
This work studies the joint design of precoding and backhaul compression strategies for the downlink of cloud radio access networks. In these systems, a central encoder is connected to multiple multiantenna base stations (BSs) via finite-capacity backhaul links. At the central encoder, precoding is followed by compression in order to produce the rate-limited bit streams delivered to each BS over the corresponding backhaul link. In current state-of-the-art approaches, the signals intended for different BSs are compressed independently. In contrast, this work proposes to leverage joint compression, also referred to as multivariate compression, of the signals of different BSs in order to better control the effect of the additive quantization noises at the mobile stations (MSs). The problem of maximizing the weighted sumrate with respect to both the precoding matrix and the joint correlation matrix of the quantization noises is formulated subject to power and backhaul capacity constraints. An iterative algorithm is proposed that achieves a stationary point of the problem. Moreover, in order to enable the practical implementation of multivariate compression across BSs, a novel architecture is proposed based on successive steps of minimum mean-squared error (MMSE) estimation and per-BS compression. Robust design with respect to imperfect channel state information is also discussed. From numerical results, it is confirmed that the proposed joint precoding and compression strategy outperforms conventional approaches based on the separate design of precoding and compression or independent compression across the BSs.
\end{abstract}

S.-H. Park and O. Simeone are with the Center for Wireless Communications and Signal Processing Research (CWCSPR), ECE Department, New Jersey Institute of Technology (NJIT), Newark, NJ 07102, USA (email: \{seok-hwan.park, osvaldo.simeone\}@njit.edu).

O. Sahin is with InterDigital Inc., Melville, New York, 11747, USA (email: Onur.Sahin@interdigital.com).

S. Shamai (Shitz) is with the Department of Electrical Engineering, Technion, Haifa, 32000, Israel (email: sshlomo@ee.technion.ac.il). 


\section{Index Terms}

Cloud radio access network, constrained backhaul, precoding, multivariate compression, network MIMO.

\section{INTRODUCTION}

Cellular systems are evolving into heterogeneous networks consisting of distributed base stations (BSs) covering overlapping areas of different sizes, and thus the problems of interference management and cell association are becoming complicated and challenging [1]. One of the most promising solutions to these problems is given by so called cloud radio access networks, in which the encoding/decoding functionalities of the BSs are migrated to a central unit. This is done by operating the BSs as "soft" relays that interface with the central unit via backhaul links used to carry only baseband signals (and not "hard" data information) [2]-[8]. Cloud radio access networks are expected not only to effectively handle the inter-cell interference but also to lower system cost related to the deployment and management of the BSs. However, one of the main impairments to the implementation of cloud radio access networks is given by the capacity limitations of the digital backhaul links connecting the BSs and the central unit. These limitations are especially pronounced for pico/femto-BSs, whose connectivity is often afforded by last-mile cables [1][9], and for BSs using wireless backhaul links [10].

In the uplink of cloud radio access networks, each BS compresses its received signal to the central unit via its finite-capacity backhaul link. The central unit then performs joint decoding of all the mobile stations (MSs) based on all received compressed signals 1 . Recent theoretical results have shown that distributed compression schemes [12] can provide significant advantages over the conventional approach based on independent compression at the BSs. This is because the signals received by different BSs are statistically correlated [13]-[17], and hence distributed source coding enables the quality of the compressed signal received from one BS to be improved by leveraging the signals received from the other BSs as side information. Note that the correlation among the signals received by the BSs is particularly pronounced for systems with many small cells concentrated in given areas. While current implementations [3][18] employ conventional

\footnotetext{
${ }^{1}$ In fact, joint decompression and decoding, an approach that is now often seen as an instance of noisy network coding [11], is generally advantageous [13].
} 
independent compression across the BSs, the advantages of distributed source coding were first demonstrated in [13], and then studied in more general settings in [14]-[17]. Related works based on the idea of computing a function of the transmitted codewords at the BSs, also known as compute-and-forward, can be found in [19][20].

In the downlink of cloud radio access networks, the central encoder performs joint encoding of the messages intended for the MSs. Then, it independently compresses the produced baseband signals to be transmitted by each BS. These baseband signals are delivered via the backhaul links to the corresponding BSs, which simply upconvert and transmit them through their antennas. This system was studied in [21][22]. In particular, in [21], the central encoder performs dirty-paper coding (DPC) [23] of all MSs' signals before compression. A similar approach was studied in [22] by accounting for the effect of imperfect channel state information (CSI). Reference [24] instead proposes strategies based on compute-and-forward, showing advantages in the lowbackhaul capacity regime and high sensitivity of the performance to the channel parameters. For a review of more conventional strategies in which the backhaul links are used to convey message information, rather than the compressed baseband signals, we refer to [25]-[28].

\section{A. Contributions}

In this work, we propose a novel approach for the compression on the backhaul links of cloud radio access networks in the downlink that can be seen as the counterpart of the distributed source coding strategy studied in [14]-[17] for the uplink. Moreover, we propose the joint design of precoding and compression. A key idea is that of allowing the quantization noise signals corresponding to different BSs to be correlated with each other. The motivation behind this choice is the fact that a proper design of the correlation of the quantization noises across the BSs can be beneficial in limiting the effect of the resulting quantization noise seen at the MSs. In order to create such correlation, we propose to jointly compress the baseband signals to be delivered over the backhaul links using so called multivariate compression [12, Ch. 9]. We also show that, in practice, multivariate compression can be implemented without resorting to joint compression across all BSs, but using instead a successive compression strategy based on a sequence of Minimum Mean Squared Error (MMSE) estimation and per-BS compression steps.

After reviewing some preliminaries on multivariate compression in Sec. III, we formulate the problem of jointly optimizing the precoding matrix and the correlation matrix of the quantization 
noises with the aim of maximizing the weighted sum-rate subject to power and the backhaul constraints resulting from multivariate compression in Sec. IV. There, we also introduce the proposed architecture based on successive per-BS steps. We then provide an iterative algorithm that achieves a stationary point of the problem in Sec. $\nabla$. Moreover, we compare the proposed joint design with the more conventional approaches based on independent backhaul compression [21]-[24] or on the separate design of precoding and (multivariate) quantization in Sec. VI] The robust design with respect to imperfect CSI is also discussed in detail. In Sec. VII, extensive numerical results are provided to illustrate the advantages offered by the proposed approach. The paper is terminated with the conclusion in Sec. VIII.

Notation: We adopt standard information-theoretic definitions for the mutual information $I(X ; Y)$ between the random variables $X$ and $Y$, conditional mutual information $I(X ; Y \mid Z)$ between $X$ and $Y$ conditioned on random variable $Z$, differential entropy $h(X)$ of $X$ and conditional differential entropy $h(X \mid Y)$ of $X$ conditioned on $Y$ [12]. The distribution of a random variable $X$ is denoted by $p(x)$ and the conditional distribution of $X$ conditioned on $Y$ is represented by $p(x \mid y)$. All logarithms are in base two unless specified. The circularly symmetric complex Gaussian distribution with mean $\boldsymbol{\mu}$ and covariance matrix $\mathbf{R}$ is denoted by $\mathcal{C N}(\boldsymbol{\mu}, \mathbf{R})$. The set of all $M \times N$ complex matrices is denoted by $\mathbb{C}^{M \times N}$, and $\mathbb{E}(\cdot)$ represents the expectation operator. We use the notations $\mathbf{X} \succeq \mathbf{0}$ and $\mathbf{X} \succ \mathbf{0}$ to indicate that the matrix $\mathbf{X}$ is positive semidefinite and positive definite, respectively. Given a sequence $X_{1}, \ldots, X_{m}$, we define a set $X_{\mathcal{S}}=\left\{X_{j} \mid j \in \mathcal{S}\right\}$ for a subset $\mathcal{S} \subseteq\{1, \ldots, m\}$. The operation $(\cdot)^{\dagger}$ denotes Hermitian transpose of a matrix or vector, and notation $\Sigma_{\mathbf{x}}$ is used for the correlation matrix of random vector $\mathbf{x}$, i.e., $\Sigma_{\mathbf{x}}=\mathbb{E}\left[\mathbf{x x}^{\dagger}\right] ; \Sigma_{\mathbf{x}, \mathbf{y}}$ represents the cross-correlation matrix $\Sigma_{\mathbf{x}, \mathbf{y}}=\mathbb{E}\left[\mathbf{x y} \mathbf{y}^{\dagger}\right] ; \Sigma_{\mathbf{x} \mid \mathbf{y}}$ is used for the conditional correlation matrix, i.e., $\boldsymbol{\Sigma}_{\mathbf{x} \mid \mathbf{y}}=\mathbb{E}\left[\mathbf{x x}^{\dagger} \mid \mathbf{y}\right]$.

\section{SySTEM MODEL}

We consider the downlink of a cloud radio access network as illustrated in Fig. 1, In the system, a central encoder communicates to $N_{M}$ MSs through $N_{B}$ distributed BSs. The message $M_{k}$ for each $k$ th $\mathrm{MS}$ is uniformly distributed in the set $\left\{1, \ldots, 2^{n R_{k}}\right\}$, where $n$ is blocklength and $R_{k}$ is the information rate of message $M_{k}$ in bits per channel use (c.u.). Each MS $k$ has $n_{M, k}$ receive antennas for $k=1, \ldots, N_{M}$, and each BS $i$ is equipped with $n_{B, i}$ antennas for $i=1, \ldots, N_{B}$. Note that the BSs can be either macro-BSs or pico/femto-BSs and that the MSs 


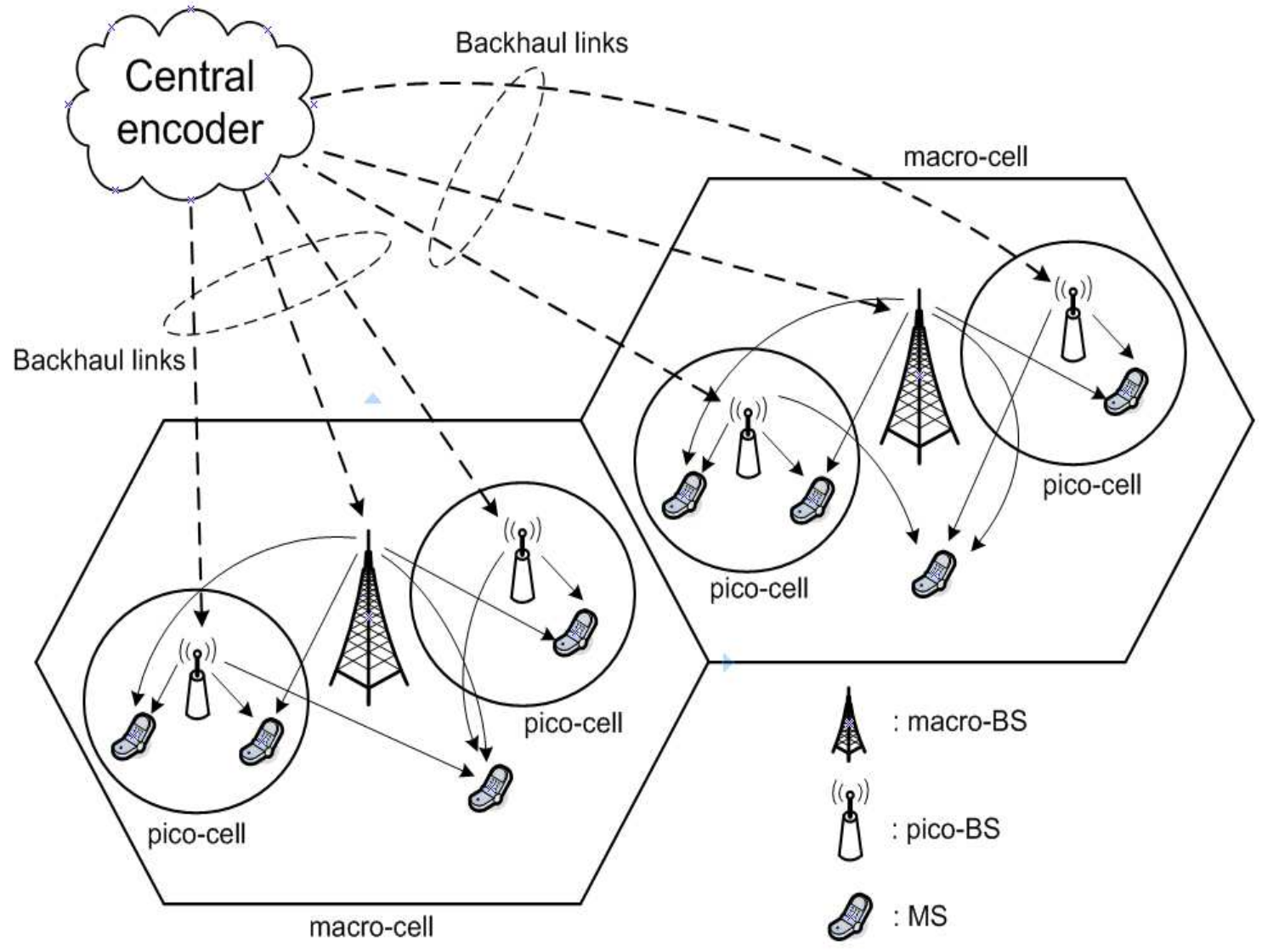

Figure 1. Downlink communication in a cloud radio access network in which there are $N_{B}$ multi-antenna BSs and $N_{M}$ multi-antenna MSs. The $N_{B}$ BSs include both macro-BSs and pico/femto-BSs. The $N_{M}$ MSs are distributed across all the cells.

are arbitrarily distributed across the cells. Each $i$ th $\mathrm{BS}$ is connected to the central encoder via digital backhaul link with finite-capacity $C_{i}$ bits per c.u. For notational convenience, we define $n_{B}=\sum_{i=1}^{N_{B}} n_{B, i}$ as the total number of transmitting antennas, $n_{M}=\sum_{k=1}^{N_{M}} n_{M, k}$ as the total number of receive antennas, and the sets $\mathcal{N}_{\mathcal{B}}=\left\{1, \ldots, N_{B}\right\}$ and $\mathcal{N}_{\mathcal{M}}=\left\{1, \ldots, N_{M}\right\}$.

As shown in Fig. 3, each message $M_{k}$ is first encoded by a separate channel encoder, which produces a coded signal $\mathbf{s}_{k}$. The signal $\mathbf{s}_{k} \in \mathbb{C}^{r_{k} \times 1}$ corresponds to the $r_{k} \times 1$ vector of encoded symbols intended for the $k$ th MS for a given c.u., and we have $r_{k} \leq n_{M, k}$. We assume that each coded symbol $\mathbf{s}_{k}$ is taken from a conventional Gaussian codebook so that we have $\mathbf{s}_{k} \sim \mathcal{C N}(\mathbf{0}, \mathbf{I})$. The signals $\mathbf{s}_{1}, \ldots, \mathbf{s}_{N_{M}}$ are further processed by the central encoder in two stages, namely precoding and compression. As is standard practice, precoding is used in order to control the interference between the data streams intended for the same MS and for different MSs. Instead, 
compression is needed in order to produce the $N_{B}$ rate-limited bit streams delivered to each BS over the corresponding backhaul link. Specifically, recall that each BS $i$ receives up to $C_{i}$ bits per c.u. on the backhaul link from the central encoder. Further discussion on precoding and compression can be found in Sec. [V].

On the basis of the bits received on the backhaul links, each BS $i$ produces a vector $\mathbf{x}_{i} \in$ $\mathbb{C}^{n_{B, i} \times 1}$ for each c.u., which is the baseband signal to be transmitted from its $n_{B, i}$ antennas. We have the per-BS power constraints 2

$$
\mathbb{E}\left[\left\|\mathbf{x}_{i}\right\|^{2}\right] \leq P_{i}, \text { for } i \in \mathcal{N}_{\mathcal{B}}
$$

Assuming flat-fading channels, the signal $\mathbf{y}_{k} \in \mathbb{C}^{n_{M, k}}$ received by MS $k$ is written as

$$
\mathbf{y}_{k}=\mathbf{H}_{k} \mathbf{x}+\mathbf{z}_{k},
$$

where we have defined the aggregate transmit signal vector $\mathbf{x}=\left[\mathbf{x}_{1}^{\dagger}, \ldots, \mathbf{x}_{N_{B}}^{\dagger}\right]^{\dagger}$, the additive noise $\mathbf{z}_{k} \sim \mathcal{C N}(\mathbf{0}, \mathbf{I}) 3$, and the channel matrix $\mathbf{H}_{k} \in \mathbb{C}^{n_{M, k} \times n_{B}}$ toward MS $k$ as

$$
\mathbf{H}_{k}=\left[\begin{array}{llll}
\mathbf{H}_{k, 1} & \mathbf{H}_{k, 2} & \cdots & \mathbf{H}_{k, N_{B}}
\end{array}\right],
$$

with $\mathbf{H}_{k, i} \in \mathbb{C}^{n_{M, k} \times n_{B, i}}$ denoting the channel matrix from BS $i$ to MS $k$. The channel matrices remain constant for the entire coding block duration. We assume that the central encoder has information about the global channel matrices $\mathbf{H}_{k}$ for all $k \in \mathcal{N}_{\mathcal{M}}$ and that each MS $k$ is only aware of the channel matrix $\mathbf{H}_{k}$. The BSs must also be informed about the compression codebooks used by the central encoder, as further detailed later. The case of imperfect CSI at the central encoder will be discussed in Sec. V-D,

Based on the definition given above and assuming single-user detection at each MS, the rates

$$
R_{k}=I\left(\mathbf{s}_{k} ; \mathbf{y}_{k}\right)
$$

can be achieved for each MS $k \in \mathcal{N}_{\mathcal{M}}$.

\footnotetext{
${ }^{2}$ The results in this paper can be immediately extended to the case with more general power constraints of the form $\mathbb{E}\left[\mathbf{x}^{\dagger} \boldsymbol{\Theta}_{l} \mathbf{x}\right] \leq$ $\delta_{l}$ for $l \in\{1, \ldots, L\}$, where the matrix $\Theta_{l}$ is a non-negative definite matrix (see, e.g., [29, Sec. II-C]).

${ }^{3}$ Correlated noise can be easily accommodated by performing whitening at each MS $k$ to obtain (2).
} 


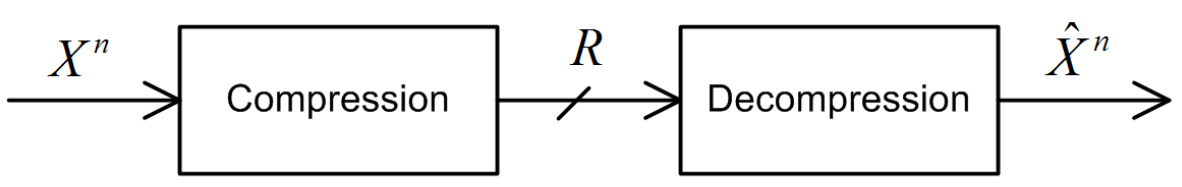

(a)

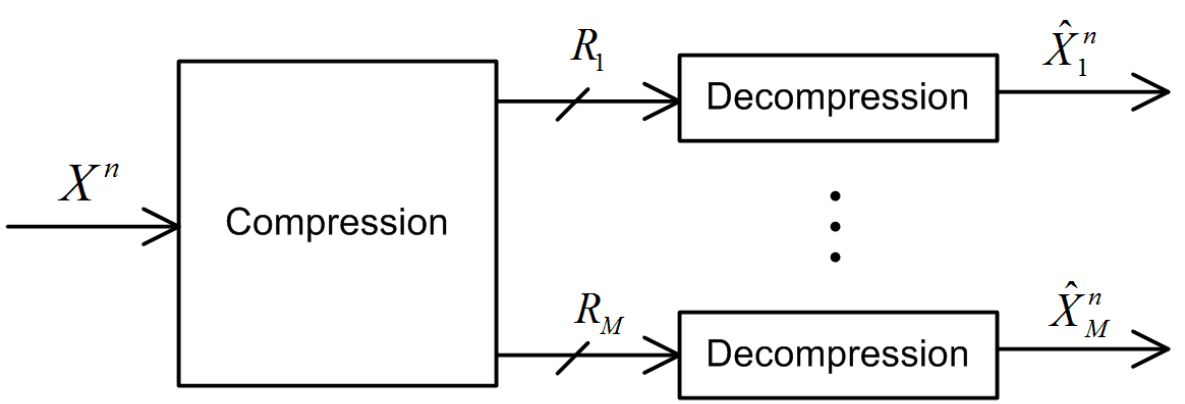

(b)

Figure 2. Illustration of $(a)$ conventional compression; $(b)$ multivariate compression.

\section{PRELIMINARIES}

This section reviews some basic information-theoretical results concerning multivariate compression, which will be leveraged in the analysis of the proposed backhaul compression strategy in Sec. IV-B,

\section{A. Conventional Compression Problem}

To fix the ideas and the notation, we first consider the conventional compression problem illustrated in Fig. 2-(a). The compression unit compresses a random sequence $X^{n}$ of $n$ independent and identically distributed (i.i.d.) samples with distribution $p(x)$ at a rate of $R$ bits per symbol. Specifically, the compressor selects a codeword $\hat{X}^{n}$ within a codebook $\mathcal{C}$ of size $2^{n R}$ and sends the corresponding index, of $n R$ bits, to the decompression unit. At the decompression unit, the sequence $\hat{X}^{n} \in \mathcal{C}$ indicated by the received index is recovered. Using the standard informationtheoretic formulation, the compression strategy is specified by a conditional distribution $p(\hat{x} \mid x)$, which is referred to as the test channel (see, e.g., [12, Ch. 2]). For a given test channel, 
compression consists in selecting a sequence $\hat{X}^{n} \in \mathcal{C}$ that is jointly typical $X^{n}$ with respect to the given joint distribution $p(x, \hat{x})=p(x) p(\hat{x} \mid x)$. Compression is hence successful if the encoder is able to find a jointly typical sequence $\hat{X}^{n}$ in the codebook $\mathcal{C}$. A classical result in information theory is that this happens with arbitrarily large probability as the block length $n$ grows large if the inequality

$$
I(X ; \hat{X}) \leq R
$$

is satisfied [12, Ch. 3][30].

\section{B. Multivariate Compression Problem}

We now review the more general multivariate compression illustrated in Fig. 2-(b). Here, the sequence $X^{n}$ is compressed into $M$ indices with the goal of producing correlated compressed versions $\hat{X}_{1}^{n}, \ldots, \hat{X}_{M}^{n}$. Each $i$ th index indicates a codeword within a codebook $\mathcal{C}_{i}$ of size $2^{n R_{i}}$, and is sent to the $i$ th decompression unit for $i \in\{1, \ldots, M\}$. Each $i$ th decompression unit then recovers a sequence $\hat{X}_{i}^{n} \in \mathcal{C}_{i}$ corresponding to the received index. We emphasize that the choice of the codewords $\hat{X}_{1}^{n}, \ldots, \hat{X}_{M}^{n}$ is done jointly at the compression unit. In particular, the specification of the compression strategy is given by a test channel $p\left(\hat{x}_{1}, \ldots, \hat{x}_{M} \mid x\right)$. This implies that the compression unit wishes to find codewords $\hat{X}_{1}^{n}, \ldots, \hat{X}_{M}^{n}$ that are jointly typical with the sequence $X^{n}$ with respect to the given joint distribution $p\left(x, \hat{x}_{1}, \ldots, \hat{x}_{M}\right)=p(x) p\left(\hat{x}_{1}, \ldots, \hat{x}_{M} \mid x\right)$. The following lemma provides a sufficient condition for multivariate compression to be successful (we refer to [12, Lemma 8.2] for a more precise statement).

Lemma 1. [12, Ch. 9] Consider an i.i.d. sequence $X^{n}$ and $n$ large enough. Then, there exist codebooks $\mathcal{C}_{1}, \ldots, \mathcal{C}_{M}$ with rates $R_{1}, \ldots, R_{M}$, that have at least one tuple of codewords $\left(\hat{X}_{1}^{n}, \ldots, \hat{X}_{M}^{n}\right) \in \mathcal{C}_{1} \times \ldots \times \mathcal{C}_{M}$ jointly typical with $X^{n}$ with respect to the given joint distribution $p\left(x, \hat{x}_{1}, \ldots, \hat{x}_{M}\right)=p(x) p\left(\hat{x}_{1}, \ldots, \hat{x}_{M} \mid x\right)$ with probability arbitrarily close to one, if the inequalities

$$
\sum_{i \in \mathcal{S}} h\left(\hat{X}_{i}\right)-h\left(\hat{X}_{\mathcal{S}} \mid X\right) \leq \sum_{i \in \mathcal{S}} R_{i}, \text { for all } \mathcal{S} \subseteq\{1, \ldots, M\}
$$

\footnotetext{
${ }^{4}$ Two sequences $X^{n}$ and $Y^{n}$ are called jointly typical with respect to a distribution $p(x, y)$ if their joint empirical distribution (i.e., normalized histogram with step size $\Delta \rightarrow 0$ ) does not deviate much from $p(x, y)$ (see, e.g., [12] Ch. 2] for a formal definition).
} 
are satisfied.

Proof: See [12, Ch. 9] for a proof.

We observe that, for a given test channel $p\left(\hat{x}_{1}, \ldots, \hat{x}_{M} \mid x\right)$, the inequalities (6) impose joint conditions on the rate of all codebooks. This is due to the requirement of finding codewords $\hat{X}_{1}, \ldots, \hat{X}_{M}$ that are jointly correlated according to the given test channel $p\left(\hat{x}_{1}, \ldots, \hat{x}_{M} \mid x\right)$. Also, note that the vector $X^{n}$ may be such that each $X_{i}$ is itself a vector and that the distortion requirements at each decompression unit prescribe that the decompression unit be interested in only a subset of entries in this vector. The connection between the multivariate set-up in Fig. 2-(b) and the system model under study in Fig. 1 will be detailed in the next section.

\section{Proposed Approach AND Problem Definition}

In this section, we first propose a novel precoding-compression strategy based on multivariate compression for the downlink of a cloud radio access network. We then establish the problem definition. Finally, a novel architecture that implements multivariate compression via a sequence of MMSE estimation and per-BS compression steps is proposed.

\section{A. Encoding Operation at the Central Encoder}

As mentioned in the previous section, the operation at the central encoder can be represented by the block diagram in Fig. 3. Specifically, after channel encoding, the encoded signals $\mathbf{s}=$ $\left[\mathbf{s}_{1}^{\dagger}, \ldots, \mathbf{s}_{N_{M}}^{\dagger}\right]^{\dagger}$ undergo precoding and compression, as detailed next.

1. Precoding: In order to allow for interference management both across the MSs and among the data streams for the same MS, the signals in vector $\mathbf{s}$ are linearly precoded via multiplication of a complex matrix $\mathbf{A} \in \mathbb{C}^{n_{B} \times n_{M}}$. The precoded data can be written as

$$
\tilde{\mathbf{x}}=\mathbf{A s},
$$

where the matrix $\mathbf{A}$ can be factorized as

$$
\mathbf{A}=\left[\mathbf{A}_{1} \cdots \mathbf{A}_{N_{M}}\right]
$$

with $\mathbf{A}_{k} \in \mathbb{C}^{n_{B} \times n_{M, k}}$ denoting the precoding matrix corresponding to MS $k$. The precoded data

$\tilde{\mathbf{x}}$ can be written as $\tilde{\mathbf{x}}=\left[\tilde{\mathbf{x}}_{1}^{\dagger}, \ldots, \tilde{\mathbf{x}}_{N_{B}}^{\dagger}\right]^{\dagger}$, where the signal $\tilde{\mathbf{x}}_{i}$ is the $n_{B, i} \times 1$ precoded vector corresponding to the $i$ th $\mathrm{BS}$ and given as

$$
\tilde{\mathbf{x}}_{i}=\mathbf{E}_{i}^{\dagger} \mathbf{A} \mathbf{s}
$$




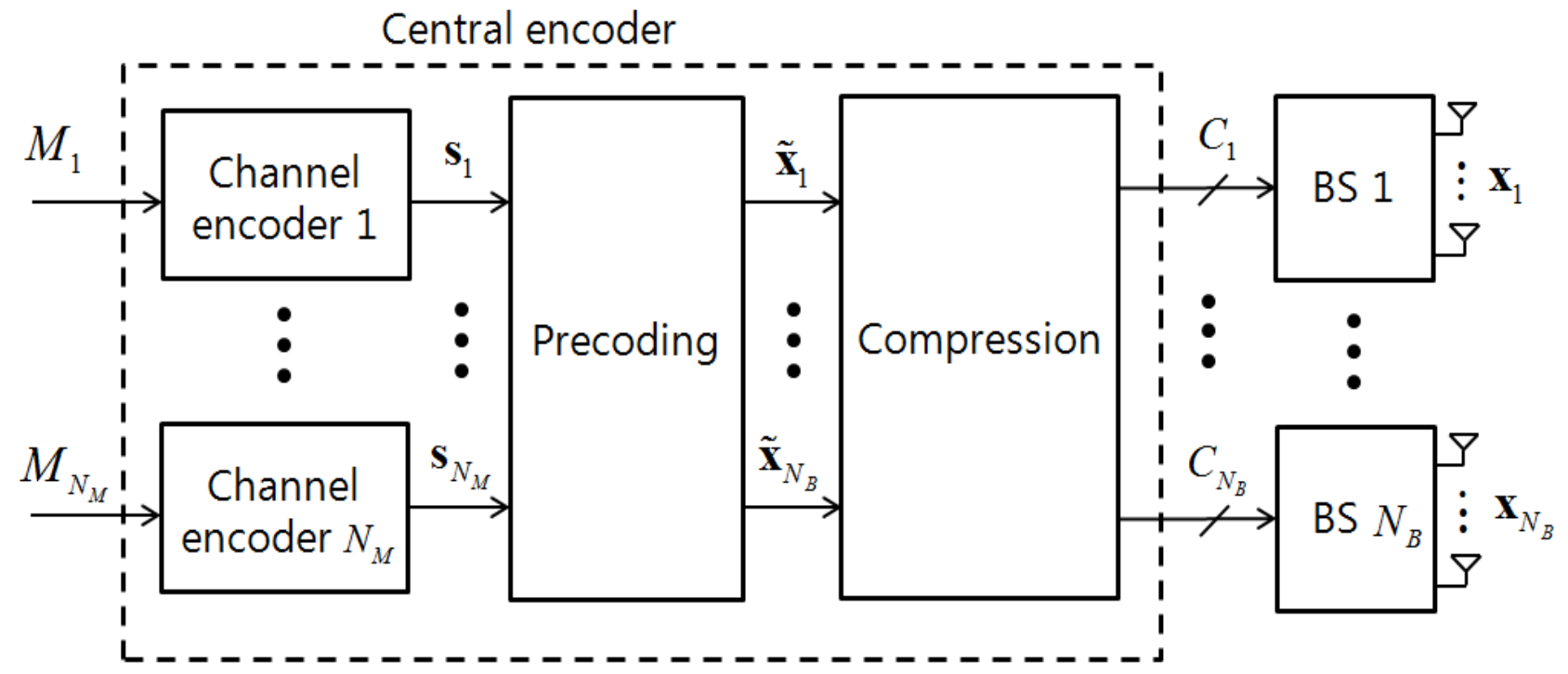

Figure 3. Illustration of the operation at the central encoder.

with the matrix $\mathbf{E}_{i} \in \mathbb{C}^{n_{B} \times n_{B, i}}$ having all zero elements except for the rows from $\left(\sum_{j=1}^{i-1} n_{B, j}+1\right)$ to $\left(\sum_{j=1}^{i} n_{B, j}\right)$ which contain an $n_{B, i} \times n_{B, i}$ identity matrix. Note that non-linear precoding using DPC techniques can also be considered, as discussed in Remark 3 below.

2. Compression: Each precoded data stream $\tilde{\mathbf{x}}_{i}$ for $i \in \mathcal{N}_{\mathcal{B}}$ must be compressed in order to allow the central encoder to deliver it to the $i$ th BS through the backhaul link of capacity $C_{i}$ bits per c.u. Each $i$ th BS then simply forwards the compressed signal $\mathbf{x}_{i}$ obtained from the central encoder. Note that this implies that the BSs need not be aware of the channel codebooks and of the precoding matrix A used by the central encoder. Instead, they must be informed about the quantization codebooks selected by the central encoder.

Using standard rate-distortion considerations, we adopt a Gaussian test channel to model the effect of compression on the backhaul link. In particular, we write the compressed signals $\mathbf{x}_{i}$ to be transmitted by $\mathrm{BS} i$ as 5

$$
\mathbf{x}_{i}=\tilde{\mathbf{x}}_{i}+\mathbf{q}_{i}
$$

where the compression noise $\mathbf{q}_{i}$ is modeled as a complex Gaussian vector distributed as $\mathcal{C N}\left(\mathbf{0}, \boldsymbol{\Omega}_{i, i}\right)$.

\footnotetext{
${ }^{5}$ The test channel $\mathbf{x}_{i}=\mathbf{B}_{i} \tilde{\mathbf{x}}_{i}+\mathbf{q}_{i}$ is seemingly more general than (10), but this can be captured by adjusting the matrix $\mathbf{A}$ in (7).
} 
Overall, the vector $\mathbf{x}=\left[\mathbf{x}_{1}^{\dagger}, \ldots, \mathbf{x}_{N_{B}}^{\dagger}\right]^{\dagger}$ of compressed signals for all the BSs is given by

$$
\mathbf{x}=\mathbf{A s}+\mathbf{q}
$$

where the compression noise $\mathbf{q}=\left[\mathbf{q}_{1}^{\dagger}, \ldots, \mathbf{q}_{N_{B}}^{\dagger}\right]^{\dagger}$ is modeled as a complex Gaussian vector distributed as $\mathbf{q} \sim \mathcal{C N}(\mathbf{0}, \Omega)$. The compression covariance $\Omega$ is given as

$$
\boldsymbol{\Omega}=\left[\begin{array}{cccc}
\boldsymbol{\Omega}_{1,1} & \boldsymbol{\Omega}_{1,2} & \cdots & \boldsymbol{\Omega}_{1, N_{B}} \\
\boldsymbol{\Omega}_{2,1} & \boldsymbol{\Omega}_{2,2} & \cdots & \boldsymbol{\Omega}_{2, N_{B}} \\
\vdots & \vdots & \ddots & \vdots \\
\boldsymbol{\Omega}_{N_{B}, 1} & \boldsymbol{\Omega}_{N_{B}, 2} & \cdots & \boldsymbol{\Omega}_{N_{B}, N_{B}}
\end{array}\right]
$$

where the matrix $\Omega_{i, j}$ is defined as $\Omega_{i, j}=\mathbb{E}\left[\mathbf{q}_{i} \mathbf{q}_{j}^{\dagger}\right]$ and defines the correlation between the quantization noises of BS $i$ and BS $j$. Rate-distortion theory guarantees that compression codebooks can be found for any given covariance matrix $\Omega \succeq \mathbf{0}$ under appropriate constraints imposed on the backhaul links' capacities. This aspect will be further discussed in Sec. [II-B,

With the described precoding and compression operations, the achievable rate (4) for MS $k$ is computed as

$$
\begin{aligned}
I\left(\mathbf{s}_{k} ; \mathbf{y}_{k}\right) & =f_{k}(\mathbf{A}, \boldsymbol{\Omega}) \\
& \triangleq \log \operatorname{det}\left(\mathbf{I}+\mathbf{H}_{k}\left(\mathbf{A} \mathbf{A}^{\dagger}+\boldsymbol{\Omega}\right) \mathbf{H}_{k}^{\dagger}\right)-\log \operatorname{det}\left(\mathbf{I}+\mathbf{H}_{k}\left(\sum_{l \in \mathcal{N}_{\mathcal{M}} \backslash\{k\}} \mathbf{A}_{l} \mathbf{A}_{l}^{\dagger}+\boldsymbol{\Omega}\right) \mathbf{H}_{k}^{\dagger}\right) .
\end{aligned}
$$

Remark 1. In the conventional approach studied in [21]-[24], the signals $\tilde{\mathbf{x}}_{i}$ corresponding to each BS $i$ are compressed independently. This corresponds to setting $\boldsymbol{\Omega}_{i, j}=\mathbf{0}$ for all $i \neq j$ in (12). A key contribution of this work is the proposal to leverage correlated compression for the signals of different BSs in order to better control the effect of the additive quantization noises at the MSs.

Remark 2. The design of the precoding matrix $\mathbf{A}$ and of the quantization covariance $\Omega$ can be either performed separately, e.g., by using a conventional precoder A such as zero-forcing (ZF) or MMSE precoding (see, e.g., [31]-[34]), or jointly. Both approaches will be investigated in the following.

Remark 3. If non-linear precoding via DPC [23] is deployed at the central encoder with a specific encoding permutation $\pi_{\mathrm{DPC}}: \mathcal{N}_{\mathcal{M}} \rightarrow \mathcal{N}_{\mathcal{M}}$ of the MS indices $\mathcal{N}_{\mathcal{M}}$, the achievable rate $R_{\pi_{\mathrm{DPC}}(k)}$ 
for MS $\pi_{\mathrm{DPC}}(k)$ is given as $R_{\pi_{\mathrm{DPC}}(k)}=I\left(\mathbf{s}_{\pi_{\mathrm{DPC}}(k)} ; \mathbf{y}_{\pi_{\mathrm{DPC}}(k)} \mid \mathbf{s}_{\pi_{\mathrm{DPC}}(1)}, \ldots, \mathbf{s}_{\pi_{\mathrm{DPC}}(k-1)}\right)$ in lieu of (4) and can be calculated as $R_{\pi_{\mathrm{DPC}}(k)}=\tilde{f}_{\pi_{\mathrm{DPC}}(k)}(\mathbf{A}, \Omega)$ with the function $\tilde{f}_{\pi_{\mathrm{DPC}}(k)}(\mathbf{A}, \Omega)$ given as

$$
\begin{aligned}
\tilde{f}_{\pi_{\mathrm{DPC}}(k)}(\mathbf{A}, \boldsymbol{\Omega}) & \triangleq \log \operatorname{det}\left(\mathbf{I}+\mathbf{H}_{\pi_{\mathrm{DPC}}(k)}\left(\sum_{l=k}^{N_{M}} \mathbf{A}_{\pi_{\mathrm{DPC}}(l)} \mathbf{A}_{\pi_{\mathrm{DPC}}(l)}^{\dagger}+\boldsymbol{\Omega}\right) \mathbf{H}_{\pi_{\mathrm{DPC}}(k)}^{\dagger}\right) \\
& -\log \operatorname{det}\left(\mathbf{I}+\mathbf{H}_{\pi_{\mathrm{DPC}}(k)}\left(\sum_{l=k+1}^{N_{M}} \mathbf{A}_{\pi_{\mathrm{DPC}}(l)} \mathbf{A}_{\pi_{\mathrm{DPC}}(l)}^{\dagger}+\boldsymbol{\Omega}\right) \mathbf{H}_{\pi_{\mathrm{DPC}}(k)}^{\dagger}\right) .
\end{aligned}
$$

Note that the DPC is designed based on the knowledge of the noise levels (including the quantization noise) in order to properly select the MMSE scaling factor [35].

\section{B. Multivariate Backhaul Compression}

As explained above, due to the fact that the BSs are connected to the central encoder via finite-capacity backhaul links, the precoded signals $\tilde{\mathbf{x}}_{i}$ in (9) for $i \in \mathcal{N}_{\mathcal{B}}$ are compressed before they are communicated to the BSs using the Gaussian test channels (10). In the conventional case in which the compression noise signals related to the different BSs are uncorrelated, i.e., $\Omega_{i, j}=\mathbf{0}$ for all $i \neq j \in \mathcal{N}_{\mathcal{B}}$ as in [21]-[24], the signal $\mathbf{x}_{i}$ to be emitted from BS $i$ can be reliably communicated from the central encoder to BS $i$ if the condition

$$
I\left(\tilde{\mathbf{x}}_{i} ; \mathbf{x}_{i}\right)=\log \operatorname{det}\left(\mathbf{E}_{i}^{\dagger} \mathbf{A} \mathbf{A}^{\dagger} \mathbf{E}_{i}+\boldsymbol{\Omega}_{i, i}\right)-\log \operatorname{det}\left(\boldsymbol{\Omega}_{i, i}\right) \leq C_{i}
$$

is satisfied for $i \in \mathcal{N}_{\mathcal{B}}$. This follows from standard rate-distortion theoretic arguments (see, e.g., [30] and Sec. [II-A). We emphasize that (15) is valid under the assumption that each BS $i$ is informed about the quantization codebook used by the central encoder, as defined by the covariance matrix $\Omega_{i, i}$.

In this paper, we instead propose to introduce correlation among the compression noise signals, i.e., to set $\Omega_{i, j} \neq \mathbf{0}$ for $i \neq j$, in order to control the effect of the quantization noise at the MSs. As discussed in Sec. III, introducing correlated quantization noises calls for joint, and not independent, compression of the precoded signals of different BSs. As seen, the family of compression strategies that produce descriptions with correlated compression noises is often referred to as multivariate compression. By choosing the test channel according to (11) (see Sec. [II-B), we can leverage Lemma 1 to obtain sufficient conditions for the signal $\mathbf{x}_{i}$ to be reliably 
delivered to BS $i$ for all $i \in \mathcal{N}_{\mathcal{B}}$. In Lemma 2, we use $\mathbf{E}_{\mathcal{S}}$ to denote the matrix obtained by stacking the matrices $\mathbf{E}_{i}$ for $i \in \mathcal{S}$ horizontally.

Lemma 2. The signals $\mathrm{x}_{1}, \ldots, \mathbf{x}_{N_{B}}$ obtained via the test channel (11) can be reliably transferred to the BSs on the backhaul links if the condition

$$
\begin{aligned}
g_{\mathcal{S}}(\mathbf{A}, \boldsymbol{\Omega}) & \triangleq \sum_{i \in \mathcal{S}} h\left(\mathbf{x}_{i}\right)-h\left(\mathbf{x}_{\mathcal{S}} \mid \tilde{\mathbf{x}}\right) \\
& =\sum_{i \in \mathcal{S}} \log \operatorname{det}\left(\mathbf{E}_{i}^{\dagger} \mathbf{A} \mathbf{A}^{\dagger} \mathbf{E}_{i}+\boldsymbol{\Omega}_{i, i}\right)-\log \operatorname{det}\left(\mathbf{E}_{\mathcal{S}}^{\dagger} \mathbf{\Omega} \mathbf{E}_{\mathcal{S}}\right) \leq \sum_{i \in \mathcal{S}} C_{i}
\end{aligned}
$$

is satisfied for all subsets $\mathcal{S} \subseteq \mathcal{N}_{\mathcal{B}}$.

Proof: The proof follows by applying Lemma 1 by substituting $\tilde{\mathbf{x}}=$ As for the signal $X$ to be compressed, and $\mathbf{x}_{1}, \ldots, \mathbf{x}_{N_{B}}$ for the compressed versions $\hat{X}_{1}, \ldots, \hat{X}_{M}$.

Comparing (15) with (16) shows that the introduction of correlation among the quantization noises for different BSs leads to additional constraints on the backhaul link capacities.

\section{Weighted Sum-Rate Maximization}

Assuming the operation at the central encoder, BSs and MSs detailed above, we are interested in maximizing the weighted sum-rate $R_{\text {sum }}=\sum_{k=1}^{N_{M}} w_{k} R_{k}$ subject to the backhaul constraints (16) over the precoding matrix $\mathbf{A}$ and the compression noise covariance $\Omega$ for given weights $w_{k} \geq 0, k \in \mathcal{N}_{\mathcal{M}}$. This problem is formulated as

$$
\begin{aligned}
\underset{\mathbf{A}, \mathbf{\Omega} \succeq \mathbf{0}}{\operatorname{maximize}} & \sum_{k=1}^{N_{M}} w_{k} f_{k}(\mathbf{A}, \mathbf{\Omega}) \\
\text { s.t. } & g_{\mathcal{S}}(\mathbf{A}, \mathbf{\Omega}) \leq \sum_{i \in \mathcal{S}} C_{i}, \text { for all } \mathcal{S} \subseteq \mathcal{N}_{\mathcal{B}}, \\
& \operatorname{tr}\left(\mathbf{E}_{i}^{\dagger} \mathbf{A} \mathbf{A}^{\dagger} \mathbf{E}_{i}+\boldsymbol{\Omega}_{i, i}\right) \leq P_{i}, \text { for all } i \in \mathcal{N}_{\mathcal{B}} .
\end{aligned}
$$

The condition (17b) corresponds to the backhaul constraints due to multivariate compression as introduced in Lemma 2 , and the condition (17c) imposes the transmit power constraints (1). It is noted that the problem (17) is not easy to solve due to the non-convexity of the objective function $\sum_{k=1}^{N_{M}} w_{k} f_{k}(\mathbf{A}, \boldsymbol{\Omega})$ in (17a) and the functions $g_{\mathcal{S}}(\mathbf{A}, \boldsymbol{\Omega})$ in (17b) with respect to $(\mathbf{A}, \boldsymbol{\Omega})$. In Sec. $\mathrm{V}$, we will propose an algorithm to tackle the solution of problem (17). 


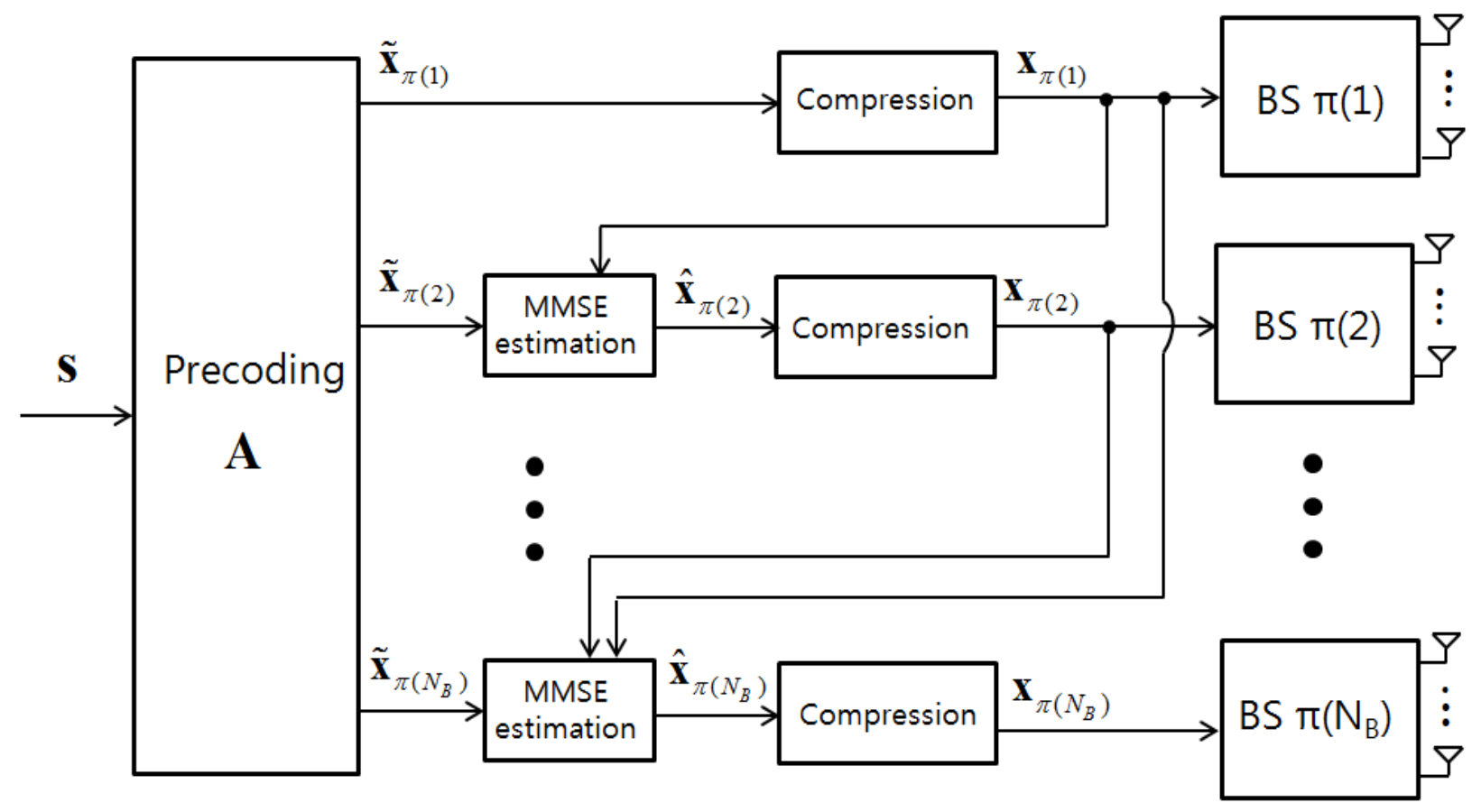

Figure 4. Proposed architecture for multivariate compression based on successive steps of MMSE estimation and per-BS compression.

\section{Successive Estimation-Compression Architecture}

In order to obtain correlated quantization noises across BSs using multivariate compression, it is in principle necessary to perform joint compression of all the precoded signals $\tilde{\mathbf{x}}_{i}$ corresponding to all BSs $i$ for $i \in \mathcal{N}_{\mathcal{B}}$ (see Sec. [III-B). If the number of BSs is large, this may easily prove to be impractical. Here, we argue that, in practice, joint compression is not necessary and that the successive strategy based on MMSE estimation and per-BS compression illustrated in Fig. 4 is sufficient. The proposed approach works with a fixed permutation $\pi: \mathcal{N}_{\mathcal{B}} \rightarrow \mathcal{N}_{\mathcal{B}}$ of the BSs' indices $\mathcal{N}_{\mathcal{B}}$.

The central encoder first compresses the signal $\tilde{\mathbf{x}}_{\pi(1)}$ using the test channel (10), namely $\mathbf{x}_{\pi(1)}=\tilde{\mathbf{x}}_{\pi(1)}+\mathbf{q}_{\pi(1)}$, with $\mathbf{q}_{\pi(1)} \sim \mathcal{C N}\left(\mathbf{0}, \Omega_{\pi(1), \pi(1)}\right)$, and sends the bit stream describing the compressed signal $\mathbf{x}_{\pi(1)}$ over the backhaul link to BS $\pi(1)$. Then, for any other $i \in \mathcal{N}_{\mathcal{B}}$ with $i>1$, the central encoder obtains the compressed signal $\mathbf{x}_{\pi(i)}$ for BS $\pi(i)$ in a successive manner in the given order $\pi$ by performing the following steps:

(a) Estimation: The central encoder obtains the MMSE estimate $\hat{\mathbf{x}}_{\pi(i)}$ of $\mathbf{x}_{\pi(i)}$ given the signal 
$\tilde{\mathbf{x}}_{\pi(i)}$ and the previously obtained compressed signals $\mathbf{x}_{\pi(1)}, \ldots, \mathbf{x}_{\pi(i-1)}$. This estimate is given by

$$
\begin{aligned}
\hat{\mathbf{x}}_{\pi(i)} & =\mathbb{E}\left[\mathbf{x}_{\pi(i)} \mid \mathbf{u}_{\pi(i)}\right] \\
& =\boldsymbol{\Sigma}_{\mathbf{x}_{\pi(i)}, \mathbf{u}_{\pi(i)}} \boldsymbol{\Sigma}_{\mathbf{u}_{\pi(i)}}^{-1} \mathbf{u}_{\pi(i)}
\end{aligned}
$$

where we defined the vector $\mathbf{u}_{\pi(i)}=\left[\mathbf{x}_{\pi(1)}^{\dagger}, \ldots, \mathbf{x}_{\pi(i-1)}^{\dagger}, \tilde{\mathbf{x}}_{\pi(i)}^{\dagger}\right]^{\dagger}$, and the correlation matrices $\Sigma_{\mathbf{x}_{\pi(i)}, \mathbf{u}_{\pi(i)}}$ and $\Sigma_{\mathbf{u}_{\pi(i)}}$ are given as

$$
\boldsymbol{\Sigma}_{\mathbf{x}_{\pi(i)}, \mathbf{u}_{\pi(i)}}=\left[\left(\mathbf{E}_{\pi(i)}^{\dagger} \mathbf{A} \mathbf{A}^{\dagger} \mathbf{E}_{\mathcal{S}_{\pi, i-1}}+\boldsymbol{\Omega}_{\pi(i), \mathcal{S}_{\pi, i-1}}\right) \mathbf{E}_{\pi(i)}^{\dagger} \mathbf{A} \mathbf{A}^{\dagger} \mathbf{E}_{\pi(i)}\right]
$$

and

$$
\Sigma_{\mathbf{u}_{\pi(i)}}=\left[\begin{array}{cc}
\mathbf{E}_{\mathcal{S}_{\pi, i-1}}^{\dagger} \mathbf{A} \mathbf{A}^{\dagger} \mathbf{E}_{\mathcal{S}_{\pi, i-1}}+\Omega_{\mathcal{S}_{\pi, i-1}, \mathcal{S}_{\pi, i-1}} & \mathbf{E}_{\mathcal{S}_{\pi, i-1}}^{\dagger} \mathbf{A A}^{\dagger} \mathbf{E}_{\pi(i)} \\
\mathbf{E}_{\pi(i)}^{\dagger} \mathbf{A A}^{\dagger} \mathbf{E}_{\mathcal{S}_{\pi, i-1}} & \mathbf{E}_{\pi(i)}^{\dagger} \mathbf{A} \mathbf{A}^{\dagger} \mathbf{E}_{\pi(i)}
\end{array}\right]
$$

with $\Omega_{\mathcal{S}, \mathcal{T}}=\mathbf{E}_{\mathcal{S}}^{\dagger} \Omega \mathbf{E}_{\mathcal{T}}$ for subsets $\mathcal{S}, \mathcal{T} \subseteq \mathcal{N}_{\mathcal{B}}$ and the set $\mathcal{S}_{\pi, i}$ defined as $\mathcal{S}_{\pi, i} \triangleq\{\pi(1), \ldots, \pi(i)\}$.

(b) Compression: The central encoder compresses the MMSE estimate $\hat{\mathbf{x}}_{\pi(i)}$ to obtain $\mathbf{x}_{\pi(i)}$ using the test channel

$$
\mathbf{x}_{\pi(i)}=\hat{\mathbf{x}}_{\pi(i)}+\hat{\mathbf{q}}_{\pi(i)},
$$

where the quantization noise $\hat{\mathbf{q}}_{\pi(i)}$ is independent of the estimate $\hat{\mathbf{x}}_{\pi(i)}$ and distributed as $\hat{\mathbf{q}}_{\pi(i)} \sim$ $\mathcal{C N}\left(\mathbf{0}, \mathbf{\Sigma}_{\mathbf{x}_{\pi(i)} \mid \hat{\mathbf{x}}_{\pi(i)}}\right)$ with

$$
\begin{aligned}
\boldsymbol{\Sigma}_{\mathbf{x}_{\pi(i)} \mid \hat{\mathbf{x}}_{\pi(i)}} & =\boldsymbol{\Sigma}_{\mathbf{x}_{\pi(i)} \mid \mathbf{u}_{\pi(i)}} \\
& =\boldsymbol{\Omega}_{\pi(i), \pi(i)}-\Omega_{\pi(i), \mathcal{S}_{\pi, i-1}} \boldsymbol{\Omega}_{\mathcal{S}_{\pi, i-1}, \mathcal{S}_{\pi, i-1}} \boldsymbol{\Omega}_{\pi(i), \mathcal{S}_{\pi, i-1}}^{\dagger} .
\end{aligned}
$$

Note that the first equality in (22) follows from the fact that the MMSE estimate $\hat{\mathbf{x}}_{\pi(i)}$ is a sufficient statistic for the estimation of $\mathbf{x}_{\pi(i)}$ from $\mathbf{u}_{\pi(i)}$ (see, e.g., [36]). Moreover, the compression rate $I\left(\hat{\mathbf{x}}_{\pi(i)} ; \mathbf{x}_{\pi(i)}\right)$ required by the test channel (21) is given by

$$
\begin{aligned}
I\left(\mathbf{x}_{\pi(i)} ; \hat{\mathbf{x}}_{\pi(i)}\right)= & h\left(\mathbf{x}_{\pi(i)}\right)-h\left(\mathbf{x}_{\pi(i)} \mid \hat{\mathbf{x}}_{\pi(i)}\right) \\
= & \log \operatorname{det}\left(\mathbf{E}_{\pi(i)}^{\dagger} \mathbf{A A}^{\dagger} \mathbf{E}_{\pi(i)}+\boldsymbol{\Omega}_{\pi(i), \pi(i)}\right) \\
& -\log \operatorname{det}\left(\boldsymbol{\Omega}_{\pi(i), \pi(i)}-\boldsymbol{\Omega}_{\pi(i), \mathcal{S}_{\pi, i-1}} \boldsymbol{\Omega}_{\mathcal{S}_{\pi, i-1}^{-1} \mathcal{S}_{\pi, i-1}} \boldsymbol{\Omega}_{\pi(i), \mathcal{S}_{\pi, i-1}}^{\dagger}\right)
\end{aligned}
$$

To see why the structure in Fig. 4 described above realizes multivariate compression, we need the following lemma. 
Lemma 3. The region of the backhaul capacity tuples $\left(C_{1}, \ldots, C_{N_{B}}\right)$ satisfying the constraints (17b) is a contrapolymatroid [37. Def. 3.1]. Therefore, it has a corner point for each permutation $\pi$ of the BS indices $\mathcal{N}_{\mathcal{B}}$, and each such corner point is given by the tuple $\left(C_{\pi(1)}, \ldots, C_{\pi\left(N_{B}\right)}\right)$ with

$$
\begin{aligned}
C_{\pi(i)} & =I\left(\mathbf{x}_{\pi(i)} ; \tilde{\mathbf{x}}, \mathbf{x}_{\pi(1)}, \ldots, \mathbf{x}_{\pi(i-1)}\right) \\
& =I\left(\mathbf{x}_{\pi(i)} ; \hat{\mathbf{x}}_{\pi(i)}\right)
\end{aligned}
$$

for $i=1, \ldots, N_{B}$. Moreover, the corner point $\left(C_{\pi(1)}, \ldots, C_{\pi\left(N_{B}\right)}\right)$ in $(24)$ is such that the constraints (17b) are satisfied with equality for the subsets $\mathcal{S}=\{\pi(1)\},\{\pi(1), \pi(2)\}, \ldots$, $\left\{\pi(1), \ldots, \pi\left(N_{B}\right)\right\}$.

Proof: This lemma follows immediately by the definition and properties of contrapolymatroids as summarized in [37, Def. 3.1]. Moreover, the second equality of (24) holds due to the fact that the MMSE estimate $\hat{\mathbf{x}}_{\pi(i)}$ is a sufficient statistic for the estimation of $\mathbf{x}_{\pi(i)}$ from $\mathbf{u}_{\pi(i)}$ (see, e.g., [36]), or equivalently from the Markov chain $\mathbf{x}_{\pi(i)}-\hat{\mathbf{x}}_{\pi(i)}-\mathbf{u}_{\pi(i)}$.

Lemma 3 shows that the region of backhaul capacities that guarantees correct delivery of the compressed signals (10) to the BSs, as identified in Lemma 2, is a type of polyhedron known as contrapolymatroid, as exemplified in Fig. 5. A specific feature of contrapolymatroid is that the corner points can be easily characterized as in (24). From standard rate-distortion theory arguments, the equality between (23) and (24) implies that the corner point $\left(C_{\pi(1)}, \ldots, C_{\pi\left(N_{B}\right)}\right)$ can be obtained for any permutation $\pi$ by the successive estimation-compression strategy outlined above and illustrated in Fig. 4,

Overall, the discussion above shows that, for any correlation matrix $\Omega$ in (12), multivariate compression is feasible by using the successive estimation-compression architecture in Fig. (4) if the backhaul capacities satisfy the corner point condition (24) for the given BS order $\pi$. Note that, in general, conditions (24) are more restrictive than (17b), which allows for any backhaul capacities in the contrapolymatroid. This is because the solution to the optimization problem (17) can be seen by contradiction to lie necessarily on the boundary of the region (17b) but

\footnotetext{
${ }^{6}$ Let us define $\mathcal{M}=\{1, \ldots, M\}$ and $f: \tilde{\mathcal{M}} \rightarrow \mathbb{R}_{+}$with $\tilde{\mathcal{M}}$ being the set of all subsets of $\mathcal{M}$. Then, the polyhedron $\left\{\left(x_{1}, \ldots, x_{M}\right) \mid \sum_{i \in \mathcal{S}} x_{i} \geq f(\mathcal{S})\right.$, for all $\left.\mathcal{S} \subseteq \mathcal{M}\right\}$ is a contrapolymatroid if the function $f$ satisfies the conditions: (a) $f(\emptyset)=0 ;(b) f(\mathcal{S}) \leq f(\mathcal{T})$ if $\mathcal{S} \subset \mathcal{T} ;(c) f(\mathcal{S})+f(\mathcal{T}) \leq f(\mathcal{S} \cup \mathcal{T})+f(\mathcal{S} \cap \mathcal{T})$ [37. Def. 3.1]
} 


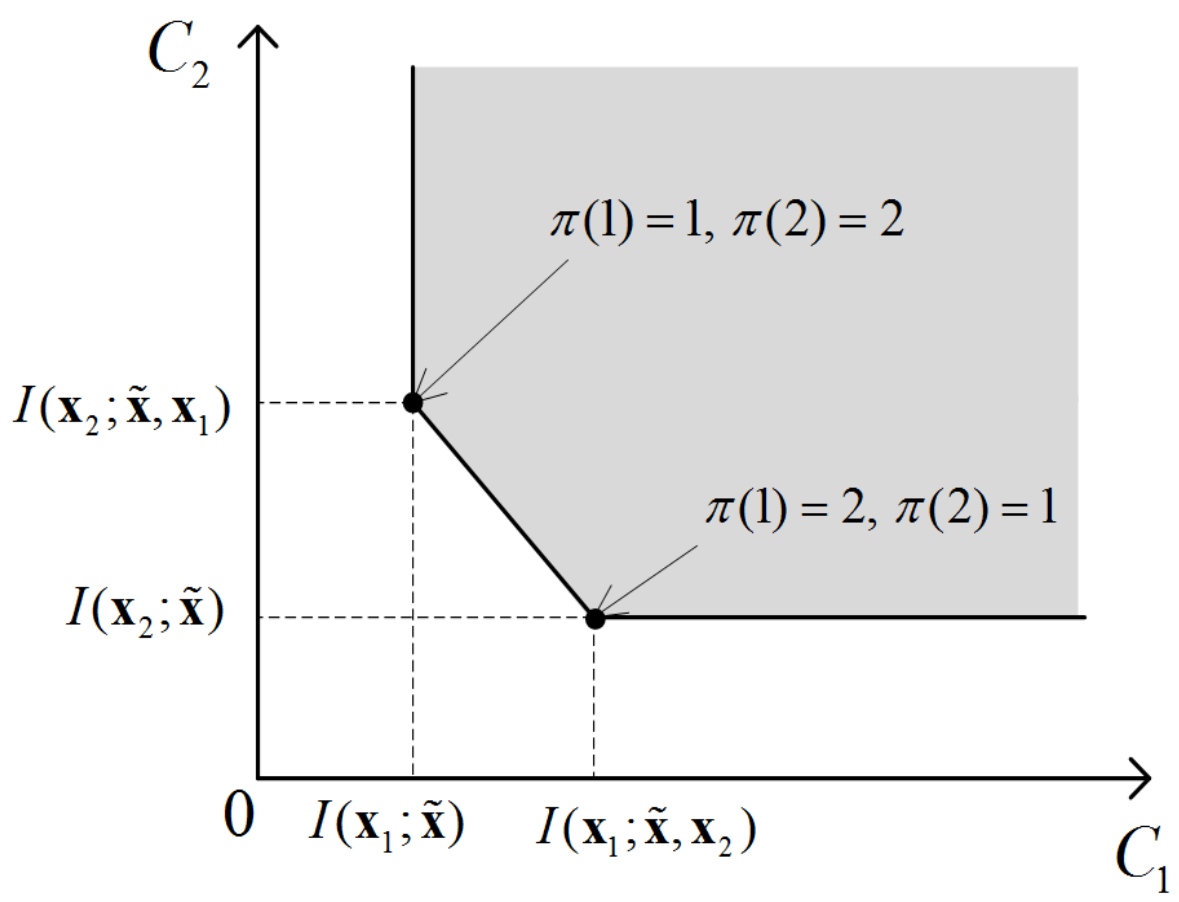

Figure 5. Illustrative example of the contrapolymatroidal region of backhaul capacities $\left(C_{1}, C_{2}\right)$ satisfying the constraint $17 \mathrm{~b}$ imposed by multivariate compression for $N_{B}=2$. The two corner points are given by (24).

possibly not on the corner points. Further discussion on this point can be found in Sec. V-B, where we observe that, in practice, this limitation is not critical.

\section{Joint Design of Precoding AND COMPRESSion}

In this section, we aim at jointly optimizing the precoding matrix $\mathbf{A}$ and the compression covariance $\Omega$ by solving problem (17). In Sec.VI, we will then consider the generally suboptimal strategy in which the precoding matrix is fixed according to standard techniques, such as ZF [31], MMSE [32] or weighted sum-rate maximizing precoding [33][34] by neglecting the compression noise, and only the compression noise matrix $\Omega$ is optimized.

\section{A. MM Algorithm}

As mentioned, the optimization (17) is a non-convex problem. To tackle this issue, we will now show how to obtain an efficient scheme that is able to achieve a stationary point of problem (17). To this end, we first make a change of variable by defining the variables $\mathbf{R}_{k} \triangleq \mathbf{A}_{k} \mathbf{A}_{k}^{\dagger}$ 
for $k \in \mathcal{N}_{\mathcal{M}}$. Then, we define the functions $f_{k}\left(\left\{\mathbf{R}_{j}\right\}_{j=1}^{N_{M}}, \boldsymbol{\Omega}\right)$ and $g_{\mathcal{S}}\left(\left\{\mathbf{R}_{k}\right\}_{k=1}^{N_{M}}, \boldsymbol{\Omega}\right)$ with respect to the variables $\left\{\mathbf{R}_{k}\right\}_{k=1}^{N_{M}}$ which are obtained by substituting $\mathbf{R}_{k}=\mathbf{A}_{k} \mathbf{A}_{k}^{\dagger}$ into the functions $f_{k}(\mathbf{A}, \Omega)$ and $g_{\mathcal{S}}(\mathbf{A}, \Omega)$ in problem (17), respectively, and the transmit power constraint as $\operatorname{tr}\left(\sum_{k=1}^{N_{M}} \mathbf{E}_{i}^{\dagger} \mathbf{R}_{k} \mathbf{E}_{i}+\boldsymbol{\Omega}_{i, i}\right) \leq P_{i}$ for $i \in \mathcal{N}_{\mathcal{B}}$. The so-obtained problem over the variables $\left\{\mathbf{R}_{k}\right\}_{k=1}^{N_{M}}$ and $\Omega$ is still non-convex due to the second term in $f_{k}\left(\left\{\mathbf{R}_{j}\right\}_{j=1}^{N_{M}, \Omega}\right)$ and the first term in $g_{\mathcal{S}}\left(\left\{\mathbf{R}_{k}\right\}_{k=1}^{N_{M}}, \boldsymbol{\Omega}\right)$, which are concave in the variables $\left\{\mathbf{R}_{k}\right\}_{k=1}^{N_{M}}$ and $\boldsymbol{\Omega}$. However, we observe that it falls into the class of difference-of-convex (DC) problems [38]. Among various algorithms having desirable properties for the solution of DC problems [38], we adopt the Majorization Minimization (MM) scheme [38], which solves a sequence of convex problems obtained by linearizing non-convex parts in the objective function $f_{k}\left(\left\{\mathbf{R}_{j}\right\}_{j=1}^{N_{M}}, \Omega\right)$ and the constraint function $g_{\mathcal{S}}\left(\left\{\mathbf{R}_{k}\right\}_{k=1}^{N_{M}}, \boldsymbol{\Omega}\right)$. It is shown that the MM algorithm converges to a stationary point of the original non-convex problems (see, e.g., [34, Theorem 1], [38, Sec. 1.3.3] and [39, Theorem 3]). The proposed algorithm is summarized in Table Algorithm 1 where we define the functions $f_{k}^{\prime}\left(\left\{\mathbf{R}_{j}^{(t+1)}\right\}_{j=1}^{N_{M}}, \boldsymbol{\Omega}^{(t+1)},\left\{\mathbf{R}_{j}^{(t)}\right\}_{j=1}^{N_{M}}, \boldsymbol{\Omega}^{(t)}\right)$ and $g_{\mathcal{S}}^{\prime}\left(\left\{\mathbf{R}_{j}^{(t+1)}\right\}_{j=1}^{N_{M}}, \boldsymbol{\Omega}^{(t+1)},\left\{\mathbf{R}_{j}^{(t)}\right\}_{j=1}^{N_{M}}, \boldsymbol{\Omega}^{(t)}\right)$ as

$$
\begin{aligned}
& f_{k}^{\prime}\left(\left\{\mathbf{R}_{j}^{(t+1)}\right\}_{j=1}^{N_{M}}, \boldsymbol{\Omega}^{(t+1)},\left\{\mathbf{R}_{j}^{(t)}\right\}_{j=1}^{N_{M}}, \boldsymbol{\Omega}^{(t)}\right) \\
\triangleq & \log \operatorname{det}\left(\mathbf{I}+\mathbf{H}_{k}\left(\sum_{j=1}^{N_{M}} \mathbf{R}_{j}^{(t+1)}+\boldsymbol{\Omega}^{(t+1)}\right) \mathbf{H}_{k}^{\dagger}\right) \\
& -\varphi\left(\mathbf{I}+\mathbf{H}_{k}\left(\sum_{j=1, j \neq k}^{N_{M}} \mathbf{R}_{j}^{(t+1)}+\boldsymbol{\Omega}^{(t+1)}\right) \mathbf{H}_{k}^{\dagger}, \mathbf{I}+\mathbf{H}_{k}\left(\sum_{j=1, j \neq k}^{N_{M}} \mathbf{R}_{j}^{(t)}+\boldsymbol{\Omega}^{(t)}\right) \mathbf{H}_{k}^{\dagger}\right)
\end{aligned}
$$

and

$$
\begin{aligned}
& g_{\mathcal{S}}^{\prime}\left(\left\{\mathbf{R}_{j}^{(t+1)}\right\}_{j=1}^{N_{M}}, \boldsymbol{\Omega}^{(t+1)},\left\{\mathbf{R}_{j}^{(t)}\right\}_{j=1}^{N_{M}}, \boldsymbol{\Omega}^{(t)}\right) \\
\triangleq & \varphi\left(\sum_{j=1}^{N_{M}} \mathbf{E}_{i}^{\dagger} \mathbf{R}_{j}^{(t+1)} \mathbf{E}_{i}+\boldsymbol{\Omega}_{i, i}^{(t+1)}, \sum_{j=1}^{N_{M}} \mathbf{E}_{i}^{\dagger} \mathbf{R}_{j}^{(t)} \mathbf{E}_{i}+\boldsymbol{\Omega}_{i, i}^{(t)}\right) \\
- & \log \operatorname{det}\left(\mathbf{E}_{\mathcal{S}}^{\dagger} \boldsymbol{\Omega}^{(t+1)} \mathbf{E}_{\mathcal{S}}\right)
\end{aligned}
$$

with the function $\varphi(\mathbf{X}, \mathbf{Y})$ given as

$$
\varphi(\mathbf{X}, \mathbf{Y}) \triangleq \log \operatorname{det}(\mathbf{Y})+\frac{1}{\ln 2} \operatorname{tr}\left(\mathbf{Y}^{-1}(\mathbf{X}-\mathbf{Y})\right) .
$$


Algorithm 1 MM Algorithm for problem (17)

1. Initialize the matrices $\left\{\mathbf{R}_{k}^{(1)}\right\}_{k=1}^{N_{M}}$ and $\boldsymbol{\Omega}^{(1)}$ to arbitrary feasible positive semidefinite matrices for problem (17) and set $t=1$.

2. Update the matrices $\left\{\mathbf{R}_{k}^{(t+1)}\right\}_{k=1}^{N_{M}}$ and $\boldsymbol{\Omega}^{(t+1)}$ as a solution of the following (convex) problem.

$$
\begin{aligned}
\underset{\left\{\mathbf{R}_{k}^{(t+1)} \succeq \mathbf{0}\right\}_{k=1}^{N_{M}}, \boldsymbol{\Omega}^{(t+1)} \succeq \mathbf{0}}{\operatorname{maximize}} & \sum_{k=1}^{N_{M}} w_{k} f_{k}^{\prime}\left(\left\{\mathbf{R}_{j}^{(t+1)}\right\}_{j=1}^{N_{M}}, \boldsymbol{\Omega}^{(t+1)},\left\{\mathbf{R}_{j}^{(t)}\right\}_{j=1}^{N_{M}}, \boldsymbol{\Omega}^{(t)}\right) \\
\text { s.t. } \quad & g_{\mathcal{S}}^{\prime}\left(\left\{\mathbf{R}_{j}^{(t+1)}\right\}_{j=1}^{N_{M}}, \boldsymbol{\Omega}^{(t+1)},\left\{\mathbf{R}_{j}^{(t)}\right\}_{j=1}^{N_{M}}, \boldsymbol{\Omega}^{(t)}\right) \leq \sum_{i \in \mathcal{S}} C_{i}, \text { for all } \mathcal{S} \subseteq \mathcal{N}_{\mathcal{B}}, \\
& \operatorname{tr}\left(\sum_{k=1}^{N_{M}} \mathbf{E}_{i}^{\dagger} \mathbf{R}_{k}^{(t+1)} \mathbf{E}_{i}+\boldsymbol{\Omega}_{i, i}^{(t+1)}\right) \leq P_{i}, \text { for all } i \in \mathcal{N}_{\mathcal{B} .} .
\end{aligned}
$$

3. Go to Step 4 if a convergence criterion is satisfied. Otherwise, set $t \leftarrow t+1$ and go back to Step 2.

4. Calculate the precoding matrices $\mathbf{A}_{k} \leftarrow \mathbf{V}_{k} \mathbf{D}_{k}^{1 / 2}$ for $k \in \mathcal{N}_{\mathcal{M}}$, where $\mathbf{D}_{k}$ is a diagonal matrix whose diagonal elements are the nonzero eigenvalues of $\mathbf{R}_{k}^{(t)}$ and the columns of $\mathbf{V}_{k}$ are the corresponding eigenvectors.

\section{B. Practical Implementation}

As we have discussed in Sec. IV given the solution $(\mathrm{A}, \Omega)$ obtained from the proposed algorithm, the central processor should generally perform joint compression in order to obtain the signals $\mathbf{x}_{i}$ to be transmitted by the BSs. However, as seen in Sec. IV-D, if the solution is such that the corner point conditions (24) are satisfied for a given permutation $\pi$ of the BSs' indices, then the simpler successive estimation-compression structure of Fig. 4 can be leveraged instead. We recall, from Lemma 3, that in order to check whether the conditions (24) are satisfied for some order $\pi$, it is sufficient to observe which inequalities (17b) are satisfied with equality: If these inequalities correspond to the subsets $\mathcal{S}=\{\pi(1)\},\{\pi(1), \pi(2)\}, \ldots,\left\{\pi(1), \ldots, \pi\left(N_{B}\right)\right\}$ for a given permutation $\pi$, then the given solution corresponds to the corner point (24) with the given $\pi$. In our extensive numerical results, we have consistently found this condition to be verified. As a result, in practice, one can implement the compression strategy characterized by the calculated covariance $\Omega$ by employing the implementation of Fig. 4 with the obtained ordering $\pi$. 


\section{Independent Quantization}

For reference, it is useful to consider the weighted sum-rate maximization problem with independent quantization noises as in [21]-[24]. This is formulated as (17) with the additional constraints

$$
\boldsymbol{\Omega}_{i, j}=\mathbf{0} \text {, for all } i \neq j \in \mathcal{N}_{\mathcal{B}}
$$

Since the constraints (29) are affine, the MM algorithm in Table Algorithm 1 is still applicable by simply setting to zero matrices $\Omega_{i, j}=\mathbf{0}$ for $i \neq j$ as per (29).

\section{Robust Design with Imperfect CSI}

So far, we have assumed that the central encoder has information about the global channel matrices $\mathbf{H}_{k}$ for $k \in \mathcal{N}_{\mathcal{M}}$. In this subsection, we discuss the robust design of the precoding matrix $\mathbf{A}$ and the compression covariance $\Omega$ in the presence of uncertainty at the central encoder regarding the channel matrices $\mathbf{H}_{k}$ for $k \in \mathcal{N}_{\mathcal{M}}$. Specifically, we focus on deterministic worstcase optimization under two different uncertainty models, namely singular value uncertainty [40] and ellipsoidal uncertainty models (see [41][42, Sec. 4.1] and references therein). While the singular value uncertainty model can be related via appropriate bounds to any normed uncertainty on the channel matrices, as discussed in [40, Sec. V], the ellipsoidal uncertainty model is more accurate when knowledge of the covariance matrix of the CSI error, due, e.g., to estimation, is available [42, Sec. 4.1]. In the following, we briefly discuss both models.

1) Singular Value Uncertainty Model: Considering multiplicative uncertainty model of [40, Sec. II-A], the actual channel matrix $\mathbf{H}_{k}$ toward each MS $k$ is modeled as

$$
\mathbf{H}_{k}=\hat{\mathbf{H}}_{k}\left(\mathbf{I}+\boldsymbol{\Delta}_{k}\right)
$$

where the matrix $\hat{\mathbf{H}}_{k}$ is the CSI known at the central encoder and the matrix $\boldsymbol{\Delta}_{k} \in \mathbb{C}^{n_{B} \times n_{B}}$ accounts for the multiplicative uncertainty matrix. The latter is bounded as

$$
\sigma_{\max }\left(\boldsymbol{\Delta}_{k}\right) \leq \epsilon_{k}<1
$$

where $\sigma_{\max }(\mathbf{X})$ is the largest singular value of matrix $\mathbf{X}$. Then, the problem of interest is to maximizing the worst-case weighted sum-rate over all possible uncertainty matrices $\boldsymbol{\Delta}_{k}$ for 
$k \in \mathcal{N}_{\mathcal{M}}$ subject to the backhaul capacity $(17 \mathrm{~b})$ and power constraints $(17 \mathrm{c})$, namely

$$
\begin{array}{cc}
\underset{\mathbf{A}, \boldsymbol{\Omega} \succeq \mathbf{0}}{\operatorname{maximize}} & \min _{\left\{\boldsymbol{\Delta}_{k} \text { s.t. }[1]\right\}_{k=1}^{N_{M}}} \sum_{k=1}^{N_{M}} w_{k} f_{k}(\mathbf{A}, \boldsymbol{\Omega}) \\
\text { s.t. } & g_{\mathcal{S}}(\mathbf{A}, \boldsymbol{\Omega}) \leq \sum_{i \in \mathcal{S}} C_{i}, \text { for all } \mathcal{S} \subseteq \mathcal{N}_{\mathcal{B}}, \\
& \operatorname{tr}\left(\mathbf{E}_{i}^{\dagger} \mathbf{A} \mathbf{A}^{\dagger} \mathbf{E}_{i}+\boldsymbol{\Omega}_{i, i}\right) \leq P_{i}, \text { for all } i \in \mathcal{N}_{\mathcal{B}} .
\end{array}
$$

The following lemma offers an equivalent formulation for problem (32).

Lemma 4. The problem (32) is equivalent to the problem (17) with the channel matrix $\mathbf{H}_{k}$ replaced with $\left(1-\epsilon_{k}\right) \hat{\mathbf{H}}_{k}$ for $k \in \mathcal{N}_{\mathcal{M}}$.

Proof: We first observe that the uncertainty matrix $\Delta_{k}$ affects only the corresponding rate function $f_{k}(\mathbf{A}, \Omega)$ in (13). Therefore, the minimization versus matrices $\boldsymbol{\Delta}_{k}$ for $k \in \mathcal{N}_{\mathcal{M}}$ in (32a) can be performed separately for each $k$ by solving the problem $\min _{\Delta_{k}} f_{k}(\mathbf{A}, \Omega)$. It can be now easily seen, following [40, Theorem 1], that the result of this minimization is obtained when $\Delta_{k}$ is such that $\Delta_{k}=-\epsilon_{k} \mathbf{I}$. This concludes the proof.

Based on Lemma 4, one can hence solve problem (32) by using the MM algorithm in Table Algorithm 1 with only change of the channel matrices from $\left\{\mathbf{H}_{k}\right\}_{k=1}^{N_{M}}$ to $\left\{\left(1-\epsilon_{k}\right) \hat{\mathbf{H}}_{k}\right\}_{k=1}^{N_{M}}$.

2) Ellipsoidal Uncertainty Model: We now consider the ellipsoidal uncertainty model. To this end, for simplicity and following related literature (see, e.g., [42, Sec. 4.1]), we focus on multiple-input single-output (MISO) case where each MS is equipped with a single antenna, i.e., $n_{M, k}=1$ for $k \in \mathcal{N}_{\mathcal{M}}$. Thus, we denote the channel vector corresponding to each MS $k$ by $\mathbf{H}_{k}=\mathbf{h}_{k}^{\dagger} \in \mathbb{C}^{1 \times n_{B}}$. The actual channel $\mathbf{h}_{k}$ is then modeled as

$$
\mathbf{h}_{k}=\hat{\mathbf{h}}_{k}+\mathbf{e}_{k},
$$

with $\hat{\mathbf{h}}_{k}$ and $\mathbf{e}_{k}$ being the presumed CSI available at the central encoder and the CSI error, respectively. The error vector $\mathbf{e}_{k}$ is assumed to be bounded within the ellipsoidal region described as

$$
\mathbf{e}_{k}^{\dagger} \mathbf{C}_{k} \mathbf{e}_{k} \leq 1
$$

for $k \in \mathcal{N}_{\mathcal{M}}$ with the matrix $\mathbf{C}_{k} \succ \mathbf{0}$ specifying the size and shape of the ellipsoid [41]. 
Following the standard formulation, we consider here the "dual" problem of power minimization under signal-to-interference-plus-noise ratio (SINR) constraints for all MSs (see [42, Sec. 4.1] and references therein). This problem is stated as

$$
\begin{aligned}
\underset{\left\{\mathbf{R}_{k} \succeq \mathbf{0}\right\}_{k=1}^{N_{M}}, \mathbf{\Omega} \succeq \mathbf{0}}{\operatorname{minimize}} & \sum_{i=1}^{N_{B}} \mu_{i} \cdot \operatorname{tr}\left(\sum_{k=1}^{N_{M}} \mathbf{E}_{i}^{\dagger} \mathbf{R}_{k} \mathbf{E}_{i}+\boldsymbol{\Omega}_{i, i}\right) \\
\text { s.t. } & \frac{\mathbf{h}_{k}^{\dagger} \mathbf{R}_{k} \mathbf{h}_{k}}{\sum_{j \in \mathcal{N}_{\mathcal{M}} \backslash\{k\}} \mathbf{h}_{k}^{\dagger} \mathbf{R}_{j} \mathbf{h}_{k}+\mathbf{h}_{k}^{\dagger} \mathbf{\Omega} \mathbf{h}_{k}+1} \geq \Gamma_{k}, \text { for all } \mathbf{e}_{k} \text { with (34) and } k \in \mathcal{N}_{\mathcal{M}} \\
& g_{\mathcal{S}}(\mathbf{A}, \boldsymbol{\Omega}) \leq \sum_{i \in \mathcal{S}} C_{i} \text {, for all } \mathcal{S} \subseteq \mathcal{N}_{\mathcal{B}}
\end{aligned}
$$

where the coefficients $\mu_{i} \geq 0$ are arbitrary weights, $\Gamma_{k}$ is the SINR constraint for MS $k$, and we recall that we have $\mathbf{R}_{k} \triangleq \mathbf{A}_{k} \mathbf{A}_{k}^{\dagger}$ for $k \in \mathcal{N}_{\mathcal{M}}$. The problem (35) is challenging since it contains infinite number of constraints in (35b). But, following the conventional S-procedure [43, Appendix B.2], we can translate the constraints (35b) into a finite number of linear constraints by introducing auxiliary variables $\beta_{k}$ for $k \in \mathcal{N}_{\mathcal{M}}$, as discussed in the following lemma.

Lemma 5. The constraints (35b) hold if and only if there exist constants $\left\{\beta_{k} \geq 0\right\}_{k=1}^{N_{M}}$ such that the condition

$$
\left[\begin{array}{cc}
\boldsymbol{\Xi}_{k} & \boldsymbol{\Xi}_{k} \hat{\mathbf{h}}_{k} \\
\hat{\mathbf{h}}_{k}^{\dagger} \boldsymbol{\Xi}_{k} & \hat{\mathbf{h}}_{k}^{\dagger} \boldsymbol{\Xi}_{k} \hat{\mathbf{h}}_{k}-\Gamma_{k}
\end{array}\right]-\beta_{k}\left[\begin{array}{cc}
\mathbf{C}_{k} & \mathbf{0} \\
\mathbf{0} & -1
\end{array}\right] \succeq \mathbf{0}
$$

is satisfied for all $k \in \mathcal{N}_{\mathcal{M}}$ where we have defined $\boldsymbol{\Xi}_{k}=\mathbf{R}_{k}-\Gamma_{k} \sum_{j \in \mathcal{N}_{\mathcal{M}} \backslash\{k\}} \mathbf{R}_{j}-\Gamma_{k} \boldsymbol{\Omega}$ for $k \in \mathcal{N}_{\mathcal{M}}$.

Proof: It directly follows by applying the S-procedure [43, Appendix B.2].

By transforming the constraint (35b) into (36), we obtain a problem that falls again in the class of DC problems [38]. Therefore, one can easily derive the MM algorithm, similar to Table Algorithm 1, by linearizing the non-convex terms in the constraint (35c). The algorithm is guaranteed to converge to a stationary point of problem (35) (see, e.g., [34, Theorem 1], [38, Sec. 1.3.3] and [39, Theorem 3]).

\section{Separate Design of Precoding ANd Compression}

In this section, we discuss a simpler approach in which the precoding matrix $\mathbf{A}$ is fixed a priori to some standard scheme, such as ZF, MMSE or weighted sum-rate maximizing precoding, 
by neglecting the compression noise. The compression covariance $\Omega$ is then designed separately so as to maximize the weighted sum-rate.

\section{A. Selection of the Precoding Matrix}

The precoding matrix $\mathbf{A}$ is first selected according to some standard criterion [31]-[34] by neglecting the compression noise. A subtle issue arises when selecting the precoding matrix A that requires some discussion. Specifically, the design of A should be done by assuming a reduced power constraint, say $\gamma_{i} P_{i}$ for some $\gamma_{i} \in(0,1)$ for $i \in \mathcal{N}_{\mathcal{B}}$. The power offset factor $\gamma_{i} \in(0,1)$ is necessary since the final signal $\mathbf{x}_{i}$ transmitted by each BS $i$ is given by (10) and is thus the sum of the precoded signal $\mathbf{E}_{i}^{\dagger} \mathbf{A} \mathbf{s}$ and the compression noise $\mathbf{q}_{i}$. Therefore, if the power of the precoded part $\mathbf{E}_{i}^{\dagger} \mathrm{As}$ is selected to be equal to the power constraint $P_{i}$, the compression noise power would be forced to be zero. But this is possible only when the backhaul capacity grows to infinity due to (17b). As a result, in order to make the compression feasible, one needs to properly select the parameters $\gamma_{1}, \ldots, \gamma_{N_{B}}$ depending on the backhaul constraints.

\section{B. Optimization of the Compression Covariance}

Having fixed the precoding matrix $\mathbf{A}$, the problem then reduces to solving problem (17) only with respect to the compression covariance $\Omega$. The obtained problem is thus a DC problem which can be tackled via the MM algorithm described in Table Algorithm 1 by limiting the optimization at Step 2 only to matrix $\Omega$. It is observed that, as discussed above, this problem may not be feasible if the parameters $\gamma_{i}, i \in \mathcal{N}_{\mathcal{B}}$, are too large. In practice, one can set these parameters using various search strategies such as bisection.

\section{NUMERICAL RESULTS}

In this section, we present numerical results in order to investigate the advantage of the proposed approach based on multivariate compression and on the joint design of precoding and compression as compared to the conventional approaches based on independent compression across the BSs and separate design. We will focus on the sum-rate performance $R_{\text {sum }}=$ $\sum_{k \in \mathcal{N}_{\mathcal{M}}} R_{k}$ (i.e., we set $w_{k}=1$ in (17a)). We also assume that there is one MS active in each cell and we consider three cells, so that we have $N_{B}=N_{M}=3$. Every BS is subject to the same power constraint $P$ and has the same backhaul capacity $C$, i.e., $P_{i}=P$ and $C_{i}=C$ for $i \in \mathcal{N}_{\mathcal{B}}$. 


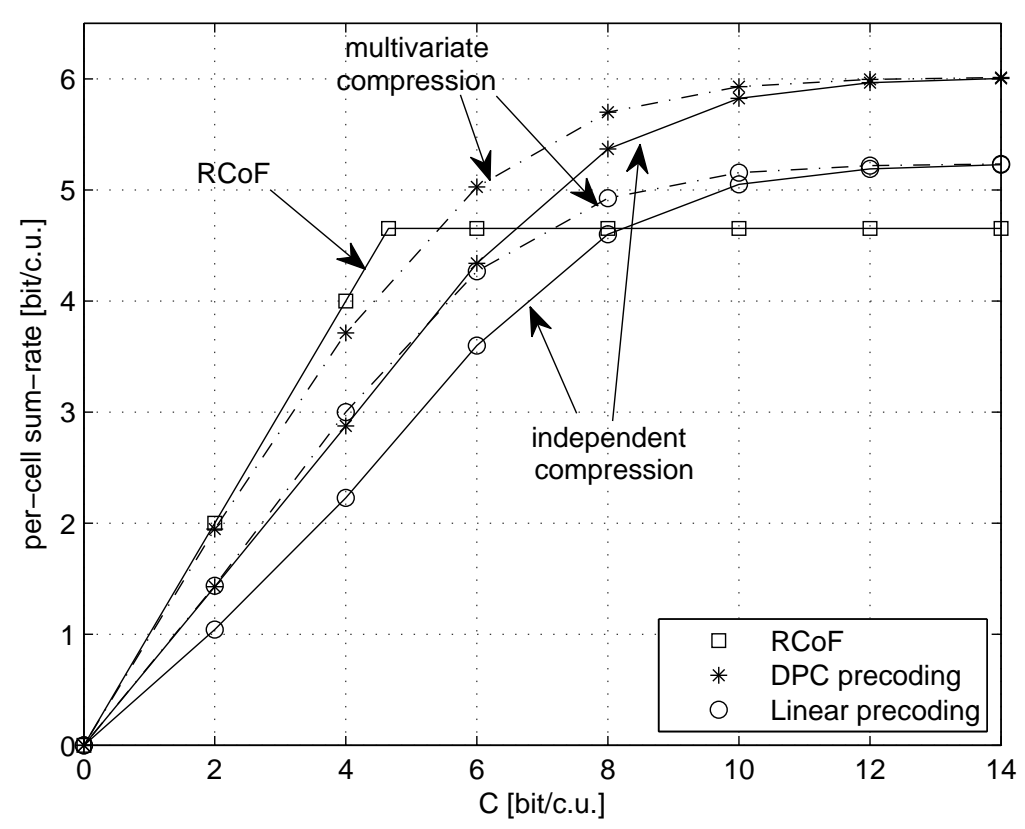

Figure 6. Per-cell sum-rate versus the backhaul capacity $C$ for the circular Wyner model [44] with $P=20 \mathrm{~dB}$ and $g=0.5$.

\section{A. Wyner Model}

We start by considering as a benchmark the performance in a simple circulant Wyner model. In this model, all MSs and BSs have a single antenna and the channel matrices $\mathbf{H}_{k, j}$ reduce to deterministic scalars given as $\mathbf{H}_{k, k}=1$ for $k=1,2,3$ and $\mathbf{H}_{k, j}=g \in[0,1]$ for $j \neq k$ [44]. In Fig. 6, we compare the proposed scheme with joint design of precoding and compression with state-of-the-art techniques, namely the compressed DPC of [21], which corresponds to using DPC precoding with independent quantization, and reverse Compute-and-Forward (RCoF) [24]. We also show the performance with linear precoding for reference. It is observed that multivariate compression significantly outperforms the conventional independent compression strategy for both linear and DPC precoding. Moreover, $\mathrm{RCoF}$ in [24] remains the most effective approach in the regime of moderate backhaul $C$, although multivariate compression allows to compensate for most of the rate loss of standard DPC precoding in the low-backhaul regime?

\footnotetext{
${ }^{7}$ The saturation of the rate of RCoF for sufficiently large $C$ is due to the integer constraints imposed on the function of the messages to be computed by the MSs [24].
} 


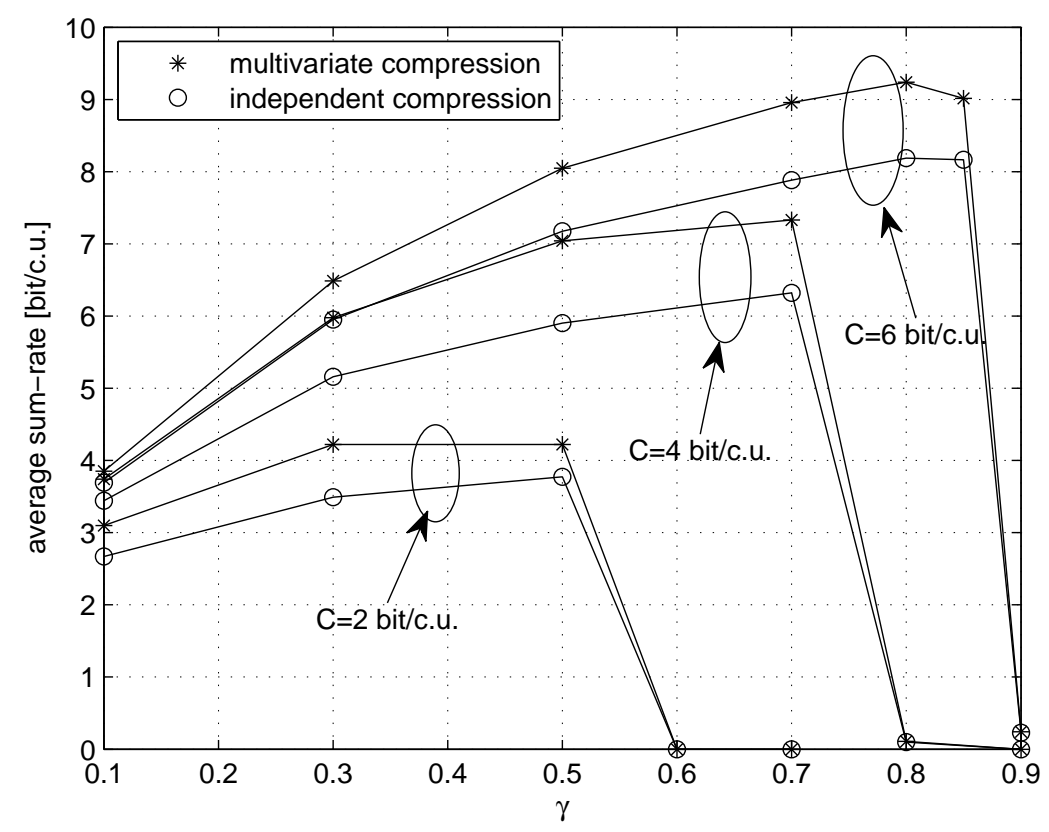

Figure 7. Average sum-rate versus the power offset factor $\gamma$ for the separate design of linear precoding and compression in Sec. $\mathrm{VI}$ with $P=5 \mathrm{~dB}$ and $\alpha=0 \mathrm{~dB}$.

\section{B. General Fading Model}

In this subsection, we evaluate the average sum-rate performance as obtained by averaging the sum-rate $R_{\text {sum }}$ over the the realization of the fading channel matrices. The elements of the channel matrix $\mathbf{H}_{k, i}$ between the MS in the $k$ th cell and the BS in the $i$ th cell are assumed to be i.i.d. complex Gaussian random variables with $\mathcal{C N}\left(0, \alpha^{|i-k|}\right)$ in which we call $\alpha$ the inter-cell channel gain. Moreover, each BS is assumed to use two transmit antennas while each MS is equipped with a single receive antenna. In the separate design, we assume that the precoding matrix $\mathbf{A}$ is obtained via the sum-rate maximization scheme in [33] under the power constraint $\gamma P$ for each BS with $\gamma \in(0,1)$ selected as discussed in Sec. VI-A Note that the algorithm of [33] finds a stationary point for the sum-rate maximization problem using the MM approach, similar to Table Algorithm 1 without consideration of the quantization noises.

Fig. 7 demonstrates the impact of the power offset factor $\gamma$ on the separate design of linear precoding and compression described in Sec. $\mathrm{VI}$ with $P=5 \mathrm{~dB}$ and $\alpha=0 \mathrm{~dB}$. Increasing $\gamma$ means that more power is available at each BS, which generally results in a better sum-rate 


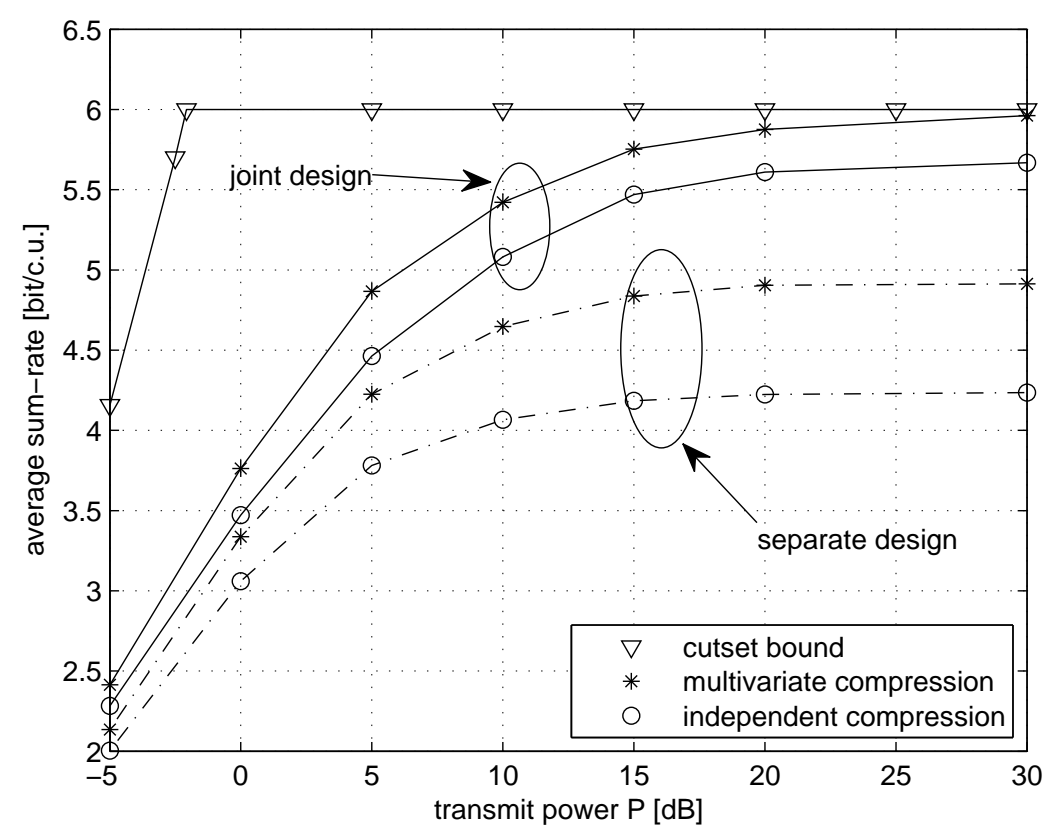

Figure 8. Average sum-rate versus the transmit power $P$ for linear precoding with $C=2$ bit/c.u. and $\alpha=0 \mathrm{~dB}$.

performance. However, if $\gamma$ exceeds some threshold value, the sum-rate is significantly degraded since the problem of optimizing the compression covariance $\Omega$ given the precoder $\mathbf{A}$ is more likely to be infeasible as argued in Sec. VI-A. This threshold value grows with the backhaul capacity, since a larger backhaul capacity allows for a smaller power of the quantization noises. Throughout the rest of this section, the power offset factor $\gamma$ is optimized via numerical search.

In Fig. 8, the average sum-rate performance of the linear precoding and compression schemes is plotted versus the transmit power $P$ with $C=2$ bit/c.u. and $\alpha=0 \mathrm{~dB}$. It is seen that the gain of multivariate compression is more pronounced when each BS uses a larger transmit power. This implies that, as the received SNR increases, more efficient compression strategies are called for. In a similar vein, the importance of the joint design of precoding and compression is more significant when the transmit power is larger. Moreover, it is seen that multivariate compression is effective in partly compensating for the suboptimality of the separate design. For reference, we also plot the cutset bound which is obtained as $\min \left\{R_{\text {full }}, 3 C\right\}$ where $R_{\text {full }}$ is the sum-capacity achievable when the BSs can fully cooperate under per-BS power constraints. We have obtained the rate $R_{\text {full }}$ by using the inner-outer iteration algorithm proposed in [45, Sec. II]. It is worth 


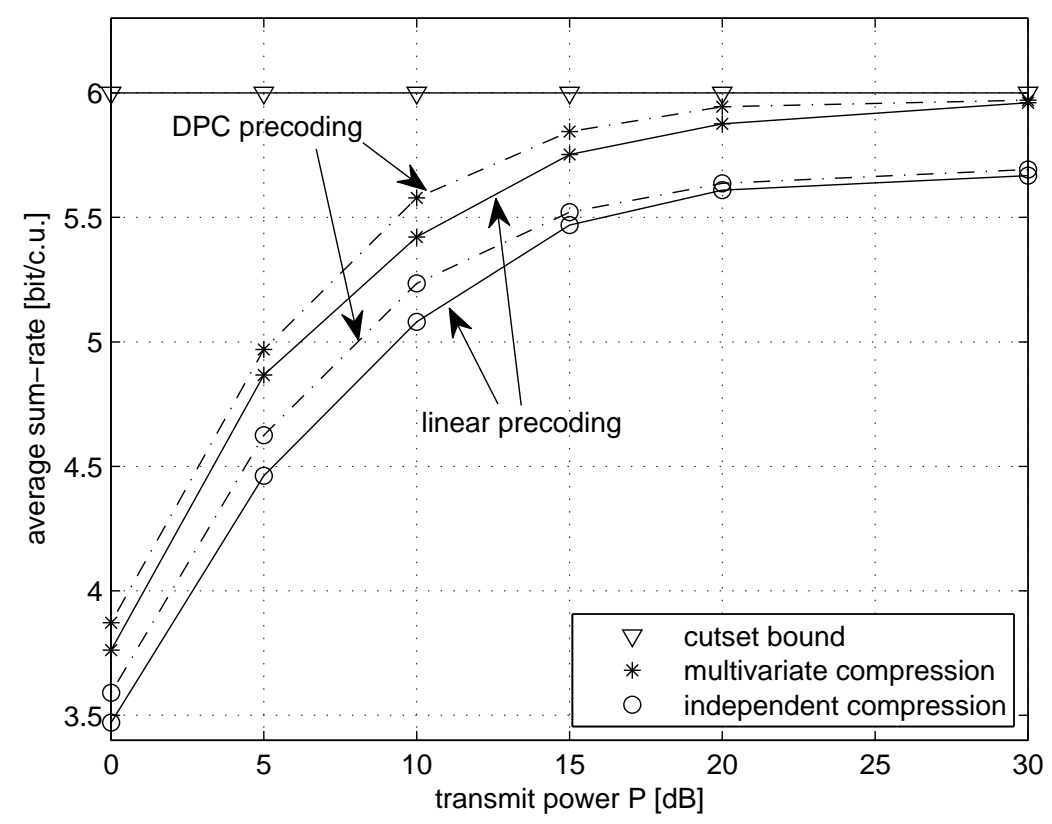

Figure 9. Average sum-rate versus the transmit power $P$ for the joint design in Sec. $\nabla$ with $C=2$ bit/c.u. and $\alpha=0 \mathrm{~dB}$.

noting that only the proposed joint design with multivariate compression approaches the cutset bound as the transmit power increases.

In Fig. 9, we compare two precoding methods, DPC and linear precoding, by plotting the average sum-rate versus the transmit power $P$ for the joint design in Sec. $\nabla$ with $C=2$ bit/c.u. and $\alpha=0 \mathrm{~dB}$. For DPC, we have applied Algorithm 1 with a proper modification for all permutations $\pi_{\mathrm{DPC}}$ of MSs' indices $\mathcal{N}_{\mathcal{M}}$ and took the largest sum-rate. Unlike the conventional broadcast channels with perfect backhaul links where there exists constant sum-rate gap between DPC and linear precoding at high SNR (see, e.g., [46]), Fig. 9 shows that DPC is advantageous only in the regime of intermediate $P$ due to the limited-capacity backhaul links. This implies that the overall performance is determined by the compression strategy rather than precoding method when the backhaul capacity is limited at high SNR.

Fig. 10 plots the average sum-rate versus the backhaul capacity $C$ for linear precoding with $P=5 \mathrm{~dB}$ and $\alpha=0 \mathrm{~dB}$. It is observed that when the backhaul links have enough capacity, the benefits of multivariate compression or joint design of precoding and compression become negligible since the overall performance becomes limited by the sum-capacity achievable when 


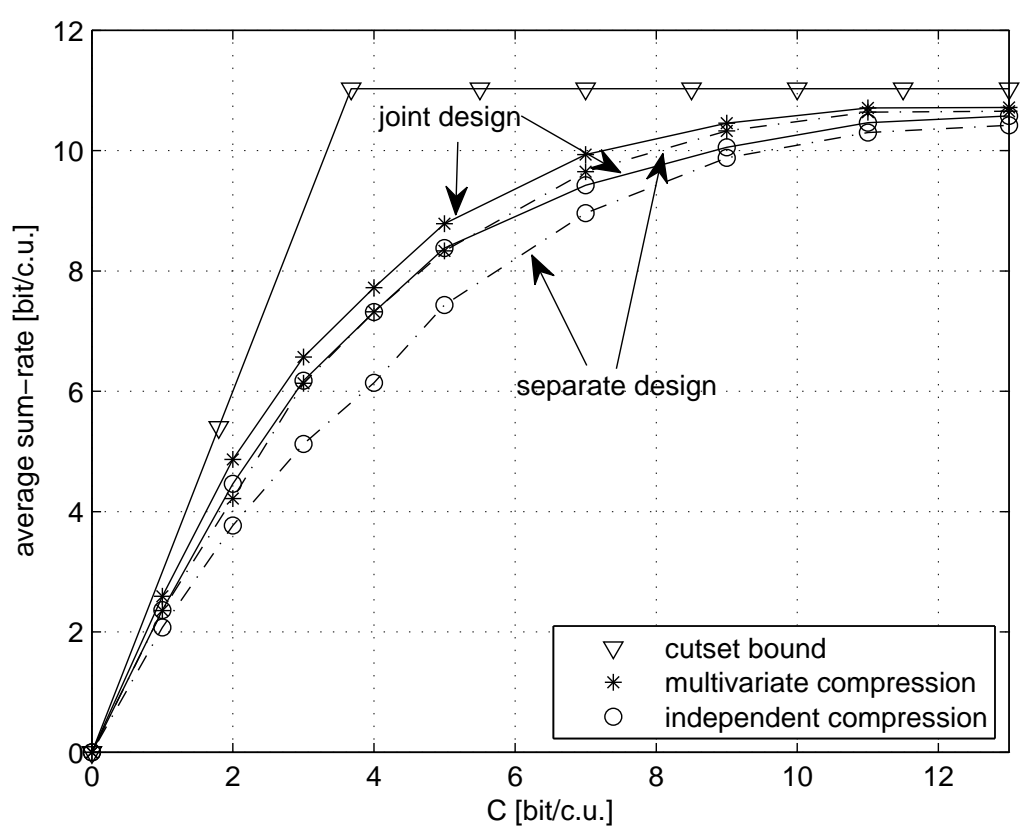

Figure 10. Average sum-rate versus the backhaul capacity $C$ for linear precoding with $P=5 \mathrm{~dB}$ and $\alpha=0 \mathrm{~dB}$.

the BSs are able to fully cooperate with each other. It is also notable that the separate design with multivariate compression outperforms the joint design with independent quantization for backhaul capacities larger than 5 bit/c.u.

Finally, we plot the sum-rate versus the inter-cell channel gain $\alpha$ for linear precoding with $C=2 \mathrm{bit} / \mathrm{c}$.u. and $P=5 \mathrm{~dB}$ in Fig. 11, We note that the multi-cell system under consideration approaches the system consisting of $N_{B}$ parallel single-cell networks as the inter-cell channel gain $\alpha$ decreases. Thus, the advantage of multivariate compression is not significant for small values of $\alpha$, since introducing correlation of the quantization noises across BSs is helpful only when each MS suffers from a superposition of quantization noises emitted from multiple BSs.

\section{CONCLUSiOnS}

In this work, we have studied the design of joint precoding and compression strategies for the downlink of cloud radio access networks where the BSs are connected to the central encoder via finite-capacity backhaul links. Unlike the conventional approaches where the signals corresponding to different BSs are compressed independently, we have proposed to exploit 


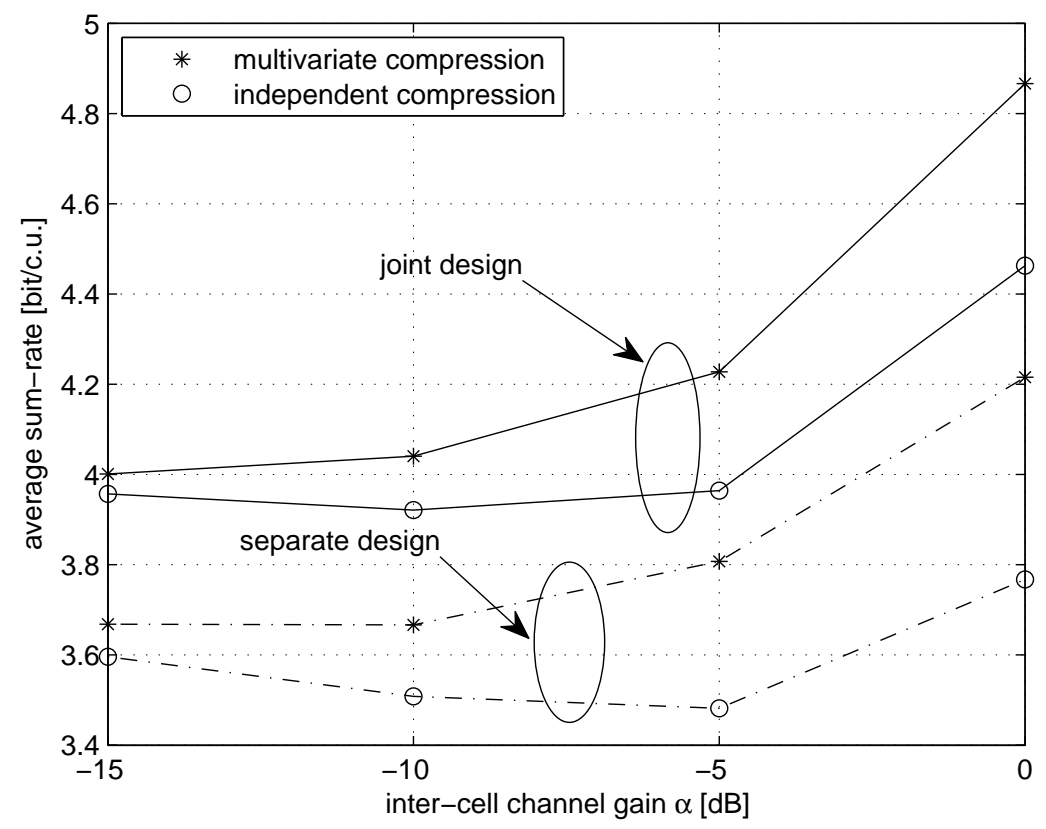

Figure 11. Average sum-rate versus the inter-cell channel gain $\alpha$ for linear precoding with $C=2$ bit/c.u. and $P=5 \mathrm{~dB}$.

multivariate compression of the signals of different BSs in order to control the effect of the additive quantization noises at the MSs. The problem of maximizing the weighted sum-rate subject to power and backhaul constraints was formulated, and an iterative MM algorithm was proposed that achieves a stationary point of the problem. Moreover, we have proposed a novel way of implementing multivariate compression that does not require joint compression of all the BSs' signals but is based on successive per-BS estimation-compression steps. Robust design with imperfect CSI was also discussed. Via numerical results, it was confirmed that the proposed approach based on multivariate compression and on joint precoding and compression strategy outperforms the conventional approaches based on independent compression and separate design of precoding and compression strategies. This is especially true when the transmit power or the inter-cell channel gain are large, and when the limitation imposed by the finite-capacity backhaul links is significant.

\section{REFERENCES}

[1] J. Andrews, "The seven ways HetNets are a paradigm shift," to appear in IEEE Comm. Mag., Mar. 2013.

[2] Intel Cor., "Intel heterogeneous network solution brief," Solution brief, Intel Core Processor, Telecommunications Industry. 
[3] J. Segel and M. Weldon, "Lightradio portfolio-technical overview," Technology White Paper 1, Alcatel-Lucent.

[4] S. Liu, J. Wu, C. H. Koh and V. K. N. Lau, "A $25 \mathrm{~Gb} / \mathrm{s}\left(/ \mathrm{km}^{2}\right)$ urban wireless network beyond IMT-advanced," IEEE Comm. Mag., pp. 122-129, Feb. 2011.

[5] China Mobile, "C-RAN: the road towards green RAN," White Paper, ver. 2.5, China Mobile Research Institute, Oct. 2011.

[6] T. Flanagan, "Creating cloud base stations with TI's keystone multicore architecture," White Paper, Texas Inst., Oct. 2011.

[7] Ericsson, "Heterogeneous networks," Ericsson White Paper, Feb. 2012.

[8] P. Marsch, B. Raaf, A. Szufarska, P. Mogensen, H. Guan, M. Farber, S. Redana, K. Pedersen and T. Kolding, "Future mobile communication networks: challenges in the design and operation," IEEE Veh. Tech. Mag., vol. 7, no. 1, pp. 16-23, Mar. 2012.

[9] V. Chandrasekhar, J. G. Andrews and A. Gatherer, "Femtocell networks: a survey," IEEE Comm. Mag., vol. 46, no. 9, pp. 59-67, Sep. 2008.

[10] I. Maric, B. Bostjancic and A. Goldsmith, "Resource allocation for constrained backhaul in picocell networks," in Proc. ITA '11, UCSD, Feb. 2011.

[11] S. H. Lim, Y.-H. Kim, A. E. Gamal and S.-Y. Chung, "Noisy network coding," IEEE Trans. Inf. Theory, vol. 57, no. 5, pp. 3132-3152, May 2011.

[12] A. E. Gamal and Y.-H. Kim, Network information theory, Cambridge University Press, 2011.

[13] A. Sanderovich, O. Somekh, H. V. Poor and S. Shamai (Shitz), "Uplink macro diversity of limited backhaul cellular network," IEEE Trans. Inf. Theory, vol. 55, no. 8, pp. 3457-3478, Aug. 2009.

[14] A. Sanderovich, S. Shamai (Shitz) and Y. Steinberg, "Distributed MIMO receiver - achievable rates and upper bounds," IEEE Trans. Inf. Theory, vol. 55, no. 10, pp. 4419-4438, Oct. 2009.

[15] A. del Coso and S. Simoens, "Distributed compression for MIMO coordinated networks with a backhaul constraint," IEEE Trans. Wireless Comm., vol. 8, no. 9, pp. 4698-4709, Sep. 2009.

[16] L. Zhou and W. Yu, "Uplink multicell processing with limited backhaul via successive interference cancellation," in Proc. IEEE Glob. Comm. Conf. (Globecom 2012), Anaheim, CA, Dec. 2012.

[17] S.-H. Park, O. Simeone, O. Sahin and S. Shamai (Shitz), "Robust and efficient distributed compression for cloud radio access networks," IEEE Trans. Veh. Tech., vol. 62, no. 2, pp. 692-703, Feb. 2013.

[18] M. Grieger, S. Boob and G. Fettweis, "Large scale field trial results on frequency domain compression for uplink joint detection," in Proc. IEEE Glob. Comm. Conf. (Globecom 2012), Anaheim, CA, Dec. 2012.

[19] B. Nazer, A. Sanderovich, M. Gastpar and S. Shamai (Shitz), "Structured superposition for backhaul constrained cellular uplink," in Proc. IEEE Intern. Sym. Inf. Theory (ISIT 2009), Seoul, Korea, Jun. 2009.

[20] S.-N. Hong and G. Caire, "Quantized compute and forward: a low-complexity architecture for distributed antenna systems," in Proc. IEEE Inf. Theory Workshop (ITW 2011), Paraty, Brazil, Oct. 2011.

[21] O. Simeone, O. Somekh, H. V. Poor and S. Shamai (Shitz), "Downlink multicell processing with limited-backhaul capacity," EURASIP J. Adv. Sig. Proc., 2009.

[22] P. Marsch and G. Fettweis, "On downlink network MIMO under a constrained backhaul and imperfect channel knowledge," in Proc. IEEE Glob. Comm. Conf. (Globecom 2009), Honolulu, Hawaii, Nov. 2009.

[23] M. H. M. Costa, "Writing on dirty paper," IEEE Trans. Inf. Theory, vol. 29, no. 3, pp. 439-441, May 1983.

[24] S.-N. Hong and G. Caire, "Reverse compute and forward: a low-complexity architecture for downlink distributed antenna systems," in Proc. IEEE Intern. Sym. Inf. Theory (ISIT 2012), Cambridge, MA, Jul. 2012. 
[25] B. L. Ng, J. S. Evans, S. V. Hanly and D. Aktas, "Distributed downlink beamforming with cooperative base stations," IEEE Trans. Inf. Theory, vol. 54, no. 12, pp. 5491-5499, Dec. 2008.

[26] I. Sohn, S. H. Lee and J. G. Andrews, "Belief propagation for distributed downlink beamforming in cooperative MIMO cellular networks," IEEE Trans. Wireless Comm., vol. 10, no. 12, pp. 4140-4149, Dec. 2011.

[27] R. Zakhour and D. Gesbert, "Optimized data sharing in multicell MIMO with finite backhaul capacity," IEEE Trans. Sig. Proc., vol. 59, no. 12, pp. 6102-6111, Dec. 2011.

[28] O. Simeone, N. Levy, A. Sanderovich, O. Somekh, B. M. Zaidel, H. V. Poor and S. Shamai (Shitz), "Cooperative wireless cellular systems: an information-theoretic view," Foundations and Trends in Communications and Information Theory, vol. 8, nos. 1-2, pp. 1-177, 2012.

[29] L. Zhang, R. Zhang, Y.-C. Liang, Y. Xin and H. V. Poor, "On Gaussian MIMO BC-MAC duality with multiple transmit covariance constraints," IEEE Trans. Inf. Theory, vol. 58, no. 4, pp. 2064-2078, Apr. 2012.

[30] X. Zhang, J. Chen, S. B. Wicker and T. Berger, "Successive coding in multiuser information theory," IEEE Trans. Inf. Theory, vol. 53, no. 6, pp. 2246-2254, Jun. 2007.

[31] R. Zhang, "Cooperative multi-cell block diagonalization with per-base-station power constraints," IEEE J. Sel. Areas Comm., vol. 28, no. 9, pp. 1435-1445, Dec. 2010.

[32] M. Hong, R.-Y. Sun, H. Baligh and Z.-Q. Luo, "Joint base station clustering and beamformer design for partial coordinated transmission in heterogeneous networks," IEEE J. Sel. Areas Comm., vol. 31, no. 2, pp. 226-240, Feb. 2013.

[33] C. T. K. Ng and H. Huang, "Linear precoding in cooperative MIMO cellular networks with limited coordination clusters," IEEE J. Sel. Areas Comm., vol. 28, no. 9, pp. 1446-1454, Dec. 2010.

[34] M. Hong, Q. Li, Y.-F. Liu and Z.-Q. Luo, "Decomposition by successive convex approximation: a unifying approach for linear transceiver design in interfering heterogeneous networks," arXiv:1210.1507

[35] U. Erez and S. t. Brink, "A close-to-capacity dirty paper coding scheme," IEEE Trans. Inf. Theory, vol. 51, no. 10, pp. 3417-3432, Oct. 2005.

[36] G. D. Forney, Jr., "Shannon meets Wiener II: On MMSE estimation in successive decoding schemes," arXiv:0409011v2.

[37] D. N. C. Tse and S. V. Hanly, "Multiaccess fading channels-Part I: polymatroid structure, optimal resource allocation and throughput capacities," IEEE Trans. Inf. Theory, vol. 44, no. 7, pp. 2796-2815, Nov. 1998.

[38] A. Beck and M. Teboulle, "Gradient-based algorithms with applications to signal recovery problems," in Convex Optimization in Signal Processing and Communications, Y. Eldar and D. Palomar, eds., pp. 42-88, Cambridge University Press. 2010.

[39] G. Scutari, F. Facchinei, P. Song, D. P. Palomar and J.-S. Pang, "Decomposition by partial linearization: parallel optimization of multi-agent systems," arXiv:1302.0756v1.

[40] S. Loyka and C. D. Charalambous, "On the compound capacity of a class of MIMO channels subject to normed uncertainty," IEEE Trans. Inf. Theory, vol. 58, no. 4, pp. 2048-2063, Apr. 2012.

[41] C. Shen, T.-H. Chang, K.-Y. Wang, Z. Qiu and C.-Y. Chi, "Distributed robust multicell coordinated beamforming with imperfect CSI: an ADMM approach," IEEE Trans. Sig. Proc., vol. 60, no. 6, pp. 2988-3003, Jun. 2012.

[42] E. Bjornson and E. Jorswieck, "Optimal resource allocation in coordinated multi-cell systems," Foundations and Trends in Communications and Information Theory, vol. 9, nos. 2-3, pp. 113-381, 2013.

[43] S. Boyd and L. Vandenberghe, Convex optimization, Cambridge University Press, 2004.

[44] D. Gesbert, S. Hanly, H. Huang, S. Shamai (Shitz), O. Simeone and W. Yu, "Multi-cell MIMO cooperative networks: a new look at interference," IEEE J. Sel. Areas Comm., vol. 28, no. 9, pp. 1380-1408, Dec. 2010. 
[45] H. Huh, H. C. Papadopoulos and G. Caire, "Multiuser MISO transmitter optimization for intercell interference mitigation," IEEE Trans. Sig. Proc., vol. 58, no. 8, pp. 4272-4285, Aug. 2010.

[46] J. Lee and N. Jindal, "High SNR analysis for MIMO broadcast channels: dirty paper coding versus linear precoding," IEEE Trans. Inf. Theory, vol. 53, no. 12, pp. 4787-4792, Dec. 2007. 